\title{
Convergence of a Three-step Iteration Scheme to the Common Fixed Points of Mixed-Type Total Asymtotically Nonexpansive Mappings in Uniformly Convex Banach Spaces
}

\author{
Imo Kalu Agwu ${ }^{1, *}$, Donatus Ikechi Igbokwe ${ }^{1}$, Nathenial C. Ukeje ${ }^{2}$ \\ ${ }^{1}$ Department of Mathematics, Micheal Okpara University of Agriculture, Umudike, Umuahia Abia State \\ Nigeria \\ agwuimo@gmail.com,igbokwedi@yahoo.com \\ ${ }^{2}$ Department of Mathematics and Statistics, University of Port-Harcourt, Port-Harcourt Rivers State \\ Nigeria \\ ukejechukwuebuanathan@gmail.com \\ *Correspondence: agwuimo@gmail.com
}

\begin{abstract}
We propose a three-step iteration scheme of hybrid mixed-type for three total asymptotically nonexpansive self mappings and three total asymptotically nonexpansive nonself mappings. In addition, we establish some weak convergence theorems of the scheme to the common fixed point of the mappings in uniformly convex Banach spaces. Our results extend and generalize numerous results currently in literature.
\end{abstract}

\section{INTRODUCTION}

Let $K$ be a nonempty subset of a real Banach space $E$. Let $T: K \longrightarrow K$ be a nonlinear mapping, we denote the set of all fixed points of $T$ by $F(T)$. The set of common fixed points of six mappings $S_{1}, S_{2}, S_{3}, T_{1}, T_{2}$ and $T_{3}$ will be denoted by $\mathcal{F}=\cap_{i=1}^{3}\left(F\left(T_{i}\right) \cap F\left(S_{i}\right)\right)$.

Definition 1.1. A mapping $T: K \longrightarrow K$ is said to asymptotically nonexpansive [6] if there exists a sequence $\left\{k_{n}\right\}$ in $[1, \infty)$ with $\lim _{n \rightarrow \infty} k_{n}=1$ such that

$$
\left\|T^{n}(x)-T^{n}(y)\right\| \leq k_{n}\|x-y\|, \forall x, y \in \mathbb{N}
$$

. In 1972, the class of asymptotically nonexpansive mapping was introduced by Goebel and Kirk [6]. They proved that if $K$ is a nonempty closed convex subset of a uniformly convex Banach space and $T$ is an asymptotically nonexpansive mapping of $K$, then $T$ has a fixed point.

Received: 29 Aug 2021.

Key words and phrases. asymtotically nonexpansive mapping; total asymptotically nonexpansive nonself mapping; hybrid mixed type iteration scheme; common fixed point; uniformly convex Banach space; weak convergence. 
Definition 1.2. A mapping $T$ is said to be total asymptotically nonexpansive [1] if

$$
\left\|T^{n}(x)-T^{n}(y)\right\| \leq\|x-y\|+\mu_{n} \phi(\|x-y\|)+\nu_{n}, \forall x, y \in K, \forall n \in \mathbb{N},
$$

where $\left\{\mu_{n}\right\}$ and $\left\{\nu_{n}\right\}$ are nonnegative real sequences such that $\mu_{n} \rightarrow 0$ and $\nu_{n} \rightarrow 0$ as $n \rightarrow \infty$ and $\phi$ is a strictly increasing continuous function $\phi:[0, \infty) \rightarrow[0, \infty)$ with $\phi(0)=0$.

From the above definitions, we see that the class of total asymptotically nonexpansive mappings includes the class of asymptotically nonexpansive mapping as a special case; see [4] for more details. Each asymptotically nonexpansive mapping is total asymptotically nonexpansive mapping with $\nu_{n}=0, \mu_{n}=k_{n}-1$ for all $n \geq 1, \phi(t)=t, t \geq 0$.

Definition 1.3. A subset $K$ of a Banach space $E$ is said to be a retract of $E$ if there exists $a$ continuous mapping $P: E \longrightarrow K$ (called retraction) such that $P(x)=x$ for all $x \in K$. If, in addition $P$ is nonexpansive, then $P$ is said to be nonexpansive retraction of $E$. If $P: E \longrightarrow K$ is a retraction, then $P^{2}=P$. A retract of a Hausdorff space must be a closed subset. Every closed convex subset of a uniformly convex Banach space is a retract.

In 2012, Yolacan and Kiziltune [18] defined the following:

Definition 1.4. Let $K$ be a nonempty and closed convex subset of a Banach space $E$. A nonself mapping $T: K \rightarrow E$ is said to be total asymptotically nonexpansive mapping if there exist sequences $k_{n}^{(1)}$ and $k_{n}^{(2)}$ in $[0, \infty)$ with $k_{n}^{(1)} \rightarrow 0$ and $k_{n}^{(2)} \rightarrow 0$ as $n \rightarrow \infty$ and a strictly increasing function $\phi:[0, \infty) \rightarrow[0, \infty)$ with $\phi(0)=0$ such that

$$
\left\|T(P T)^{n-1}(x)-T(P T)^{n-1}(y)\right\| \leq\|x-y\|+k_{(n)}^{1} \phi(\|x-y\|)+k_{n}^{(2)}, \forall x, y \in K, n \in \mathbb{N} .
$$

Chidume et al. [3] studied the following iterative scheme in 2004:

$$
\begin{aligned}
x_{1} & =x \in K \\
x_{n+1} & =P\left(\alpha_{n} T(P T)^{n-1} x_{n}+\left(1-\alpha_{n}\right) x_{n}\right), n \geq 1,
\end{aligned}
$$

where $\left\{\alpha_{n}\right\}$ is a sequence in $(0,1), K$ is a nonempty closed convex subset of of a real uniformly convex Banach space $E, P$ is a nonexpansive retraction of $E$ onto $K$, and proved some strong and weak convergence theorems for asymptotically nonexpansive nonself mappings in the intermediate sense in the framework of uniformly convex Banach spaces.

In 2006, Wang [17] generalised the iteration process (1.4) as follows:

$$
\begin{aligned}
x_{1} & =x \in K, \\
x_{n+1} & =P\left(\left(1-\alpha_{n}\right) x_{n}+\alpha_{n} T_{1}\left(P T_{1}\right)^{n-1} y_{n}\right), \\
y_{n} & =P\left(\left(1-\beta_{n}\right) x_{n}+\beta_{n} T_{2}\left(P T_{2}\right)^{n-1} x_{n}\right), n \geq 1,
\end{aligned}
$$


where $T_{1}, T_{2}: K \longrightarrow E$ are two asymptotically nonexpansive nonself mappings, $\left\{\alpha_{n}\right\}$ and $\left\{\beta_{n}\right\}$ are real sequences in $[0,1)$, and proved some weak and strong convergence theorems for asymptotically nonexpansive nonself mappings.

In 2012, Guo et al [8] generalised the iteration process (1.5) as follows:

$$
\begin{aligned}
x_{1} & =x \in K \\
x_{n+1} & =P\left(\left(1-\alpha_{n}\right) S_{1}^{n} x_{n}+\alpha_{n} T_{1}\left(P T_{1}\right)^{n-1} y_{n}\right), \\
y_{n} & =P\left(\left(1-\beta_{n}\right) S_{2}^{n} x_{n}+\beta_{n} T_{2}\left(P T_{2}\right)^{n-1} x_{n}\right), n \geq 1,
\end{aligned}
$$

where $T_{1}, T_{2}: K \longrightarrow E$ are two asymptotically nonexpansive nonself mappings, $S_{1}, S_{2}: K \longrightarrow E$ are two asymptotically nonexpansive self mappings and $\left\{\alpha_{n}\right\},\left\{\beta_{n}\right\}$ are real sequences in $[0,1)$, and proved some strong and weak convergence theorems for mixed-type asymptotically nonexpansive mappings.

\section{Hybrid Mixed-Type Iteration Scheme}

Let $E$ be a real uniformly convex Banach space, $K$ a nonempty closed convex subset of $E$ and $P: E \longrightarrow K$ a nonexpansive retraction of $E$ onto $K$. Let $S_{1}, S_{2}, S_{3}: K \longrightarrow K$ be three total asymptotically nonexpansive self mappings and $T_{1}, T_{2}, T_{3}: K \longrightarrow E$ be three total asymptotically nonexpansive nonself mappings. Then, the hybrid iteration scheme for the above mentioned mappings is as follows:

$$
\left\{\begin{array}{l}
x_{1}=x \in K \\
x_{n+1}=P\left(\left(1-\alpha_{n}\right) S_{1}^{n} x_{n}+\alpha_{n} T_{1}\left(P T_{1}\right)^{n-1} y_{n}\right) \\
y_{n}=P\left(\left(1-\beta_{n}\right) S_{2}^{n} x_{n}+\beta_{n} T_{2}\left(P T_{2}\right)^{n-1} z_{n}\right) \\
z_{n}=P\left(\left(1-\gamma_{n}\right) S_{3}^{n} x_{n}+\gamma_{n} T_{3}\left(P T_{3}\right)^{n-1} x_{n}\right)
\end{array}\right.
$$

where $\left\{\alpha_{n}\right\},\left\{\beta_{n}\right\}$, and $\left\{\gamma_{n}\right\}$ are real sequences in $[0,1)$.

The aim of this paper is to study this new hybrid mixed-type iteration scheme (1.7), prove demiclosedness principle for total asymptotically nonexpansive nonself map and establish some convergence theorems for mixed-type mappings in the setting of uniformly convex Banach spaces.

\section{Preliminary}

For the sake of convenience, we restate the following concepts and results:

Let $E$ be a Banach space with its dimension greater than or equal to 2. The modulus of convexity of $E$ is a function $\delta_{E}(\varepsilon):(0,2] \longrightarrow(0,2]$ defined by

$$
\delta_{E}(\varepsilon)=\inf \left\{1-\left\|\frac{1}{2}(x+y)\right\|:\|x\|=1,\|y\|=1, \varepsilon=\|x-y\|\right\} .
$$


A Banach space $E$ is uniformly convex if and if $\delta_{E}(\varepsilon)>0$, for all $\varepsilon \in(0,2]$.

We recall the following:

Definition 2.1. (see [19]: Let $\varrho=\{x \in E:\|x\|=1\}$ and let $E^{\star}$ be the dual of $E$. The space $E$ has Gateaux differentiable norm if $\lim _{n \rightarrow \infty} \frac{\|x+t y\|-\|x\|}{t}$ exists $\forall x, y \in \varrho$.

Definition 2.2. (see [19]: The space $E$ has Frechet differentiable norm [15] if for each $x \in \varrho$, the limit of the norm above exists and is attained uniformly for all $y \in \varrho$, and in this case, it is also well known that

$$
\langle h, J(x)\rangle+\frac{1}{2}\|x\|^{2} \leq \frac{1}{2}\|x+h\|^{2} \leq\langle h, J(x)\rangle+\frac{1}{2}\|x\|^{2}+b(\|x\|),
$$

$\forall x, y \in E$, where $J$ is the Frechet derivative of the functional $\frac{1}{2} \|\left.\cdot\right|^{2}$ at $x \in E,\langle\cdot\rangle$ is the pairing between $E$ and $E^{\star}$ and $b$ is an increasing function defined on $[0, \infty)$ such that $\lim _{t \rightarrow \infty} \frac{b(t)}{t}=0$.

Definition 2.3. : The space $E$ has Opial condition [10] if for any sequence $\left\{x_{n}\right\}$ in $E, x_{n}$ converges to $x$ weakly, then it follows that $\limsup _{n \rightarrow \infty}\left\|x_{n}-x\right\|<\limsup _{n \rightarrow \infty}\left\|x_{n}-y\right\|$ for all $y \in E$ with $x \neq y$.

Examples of Banach spaces satisfying Opial conditions are Hilbert spaces and all spaces $I^{P}(1<$ $p<\infty)$. On the other hand, $L^{p}[0, \pi]$ with $1<p \neq 2$ fails to satify Opial condition.

Definition 2.4. : A mapping $T: K \longrightarrow K$ is said to be demiclosed at 0 , if for any sequence $\left\{x_{n}\right\}$ in $K$, the condition that $x_{n}$ converges weakly to $x \in K$ and $T x_{n}$ converges strongly to 0 implies $T x=0$.

Definition 2.5. : A Banach space has the Kadec-Klec property [14] if for every sequence $x_{n}$ in $E, x_{n} \rightarrow x$ weakly and $\left\|x_{n}\right\| \rightarrow\|x\|$, then it follows that $\left\|x_{n}-x\right\| \rightarrow 0$.

Next, we state the following useful lemmas which will be needed in order to prove our main results.

Lemma 2.1. (see [16]): Let $\left\{\alpha_{n}\right\}_{n=1}^{\infty},\left\{\beta_{n}\right\}_{n=1}^{\infty}$ and $\left\{\gamma_{n}\right\}_{n=1}^{\infty}$ be sequences of nonnegative numbers satisfying the inequality:

$$
\alpha_{n+1} \leq\left(1+\beta_{n}\right) \alpha_{n}+\gamma_{n}, \forall n \geq 1
$$

If $\sum_{n=1}^{\infty} \beta_{n}<\infty$ and $\sum_{n=1}^{\infty} \gamma_{n}<\infty$, then

(1) $\lim _{n \rightarrow \infty} \alpha_{n}$ exists

(2) In particular, if $\left\{\alpha_{n}\right\}_{n=1}^{\infty}$ has a subsequence which converges strongly to 0 , then $\lim _{n \rightarrow \infty} \alpha_{n}=0$. 
Lemma 2.2. (see [14]): Let $E$ be a uniformly convex Banach space and $0<p \leq t_{n} \leq q<1$ for each $n \geq 1$. Suppose that $\left\{x_{n}\right\}$ and $\left\{y_{n}\right\}$ are sequences in $E$ such that

$$
\limsup _{n \rightarrow \infty}\left\|x_{n}\right\| \leq r, \limsup _{n \rightarrow \infty}\left\|y_{n}\right\| \leq r \text { and } \lim _{n \rightarrow \infty}\left\|t_{n} x_{n}+\left(1-t_{n}\right) y_{n}\right\|=r,
$$

hold for some $r \geq 0$. Then $\lim _{n \rightarrow \infty}\left\|x_{n}-y_{n}\right\|=0$.

Lemma 2.3. (see [14]): Let $E$ be a real reflexive Banach space such that its dual $E^{\star}$ has the Kadec-Klec property. Let $\left\{x_{n}\right\}$ be a bounded sequence in $E$ and $p, q \in \omega_{\omega}\left(x_{n}\right)$ (where $\omega_{\omega}\left(x_{n}\right)$ denotes the set of all weak subsequential limits of $\left.\left\{x_{n}\right\}\right)$. Suppose $\lim _{n \rightarrow \infty}\left\|t x_{n}+(1-t) p-q\right\|$ exists for all $t \in[0,1]$. Then, $p=q$.

Lemma 2.4. (see [14]): Let $K$ be a nonempty convex subset of a uniformly convex Banach space $E$. Then, there exists a strictly incraesing continous convex function $\phi:[0, \infty) \rightarrow[0, \infty)$ with $\phi(0)=0$ such that for each Lipshitizian mapping $T: C \longrightarrow C$ with the Lipschiz constant $L$,

$$
\|t T x-(1-t) T y-T(t x-(1-t) y)\| \leq L \phi^{-1}\left(\|x-y\|-\frac{1}{L}\|T x-T y\|\right)
$$

for all $x, y \in K$ and for all $t \in[0.1]$.

Lemma 2.5. (see [2]) Let $E$ be a uniformly convex Banach space, $K$ a nonempty bounded close convex subset of $E$. Then, there exists a strictly increasing continous convex function $\phi:[0, \infty) \longrightarrow[0, \infty)$ with $\phi(0)=0$ such that for any Lipschitizian mapping $T: K \longrightarrow E$ with Lipschitz constant $L \geq 1$ and elements $\left\{x_{n}\right\}_{j=i}^{n}$ in $K$ and any nonnegative numbers $\left\{t_{j}\right\}_{j=1}^{n}$ with $\sum_{j=1}^{n} t_{j}=1$, the following inequality holds:

$$
\left\|T\left(\sum_{j=1}^{n} t_{j} x_{j}\right)-\sum_{j=1}^{n} t_{j} T x_{j}\right\| \leq L \phi^{-1}\left\{\max _{1 \leq j, k \leq n}\left(\left\|x_{j}-x_{k}\right\|-L^{-1}\left\|T x_{j}-T x_{k}\right\|\right)\right\}
$$

Lemma 2.6. (see [21]) If the sequence $\left\{x_{n}\right\}_{n=1}^{\infty}$ converges weakly to $x$, then there exists a sequence of convex combination $y_{j}=\sum_{k=1}^{n(j)} \lambda_{k}^{(j)} x_{k+j}, \lambda_{k}^{(j)} \geq 0$ and $\sum_{k=1}^{n(j)} \lambda^{(j)}=1$, such that $\left\|y_{j}-x\right\| \rightarrow 0$. as $n \rightarrow \infty$.

\section{Main Results}

Lemma 3.1. ( Demiclosedness Principle for Nonself Total Asymptotically Nonexpansive Maps ) Let $K$ be a nonempty closed convex and bounded subset of a uniformly convex Banach space $E$ and $T: K \longrightarrow E$ be L-Lipschitz continuous and total asymptotically nonexpansive mapping with the function $\phi:[0, \infty) \longrightarrow[0, \infty)$ (such that $\phi(0)=0$ ) and nonnegative sequences $\left\{k_{n}^{(1)}\right\},\left\{k_{n}^{(2)}\right\}$ such that $k_{n}^{(1)}, k_{n}^{(2)} \rightarrow 0$ as $n \rightarrow \infty$. Then, I-T is demiclosed at 0 .

Proof. Let $\left\{x_{n}\right\}$ converge weakly to $\omega \in K$ and $\left\{x_{n}-T x_{n}\right\}$ converge strongly to 0 . We prove that $(I-T) \omega=0$. Clearly, $\left\{x_{n}\right\}$ is bounded. So, there exists $\rho>0$ such that $\left\{x_{n}\right\} \subset C=K \cap \overline{B_{\rho}}(0)$, where $\overline{B_{\rho}}(0)$ is a closed ball in $E$ with centre 0 and radius $\rho$. Thus, $C$ is nonempty, closed, 
bounded and convex subset in $K$.

Claim: $T(P T)^{n-1} \omega \rightarrow \omega$ as $n \rightarrow \infty$. In fact, since $\left\{x_{n}\right\}$ converges weakly to $\omega$, by Lemma $6($ see $[21])$, we have for all $n>1$, there exists a convex combination

$$
y_{n}=\sum_{i=1}^{m(n)} t_{i}^{(n)} x_{i+n}, t_{i}^{(n)} \geq 0 \text { and } \sum_{i=1}^{m(n)} t_{i}^{(n)}=1 \text { such that }\left\|y_{n}-\omega\right\| \rightarrow 0 \text { as } n \rightarrow \infty .
$$

Also, since $\left\{x_{n}-T x_{n}\right\}$ converges to 0 , then for any $\epsilon>0$ and a positive integer $m \geq 1$, there exists $N_{1}=N(\epsilon)>0$ such that

$$
\left\|(I-T) x_{n}\right\|<\frac{\epsilon}{1+m}, \forall n \geq N_{1} .
$$

Hence, $\forall n \geq N_{1}$, using Definition 1.4 and the fact that $P$ is nonexpansive, we have the following estimates:

For arbitrary but fixed $j \geq 1$, we have

$$
\begin{aligned}
\left\|x_{n}-T(P T)^{(j-1)} x_{n}\right\| \leq & \left\|(I-T) x_{n}\right\|+\left\|(T-T(P T)) x_{n}\right\| \\
& +\left\|\left(T(P T)-T(P T)^{2}\right) x_{n}\right\| \\
& +\left\|\left(T(P T)^{2}-T(P T)^{3}\right) x_{n}\right\| \\
& \left.+\cdots+\|\left(T(P T)^{j-2}-T(P T)^{j-1}\right)\right) x_{n} \| \\
\leq & \left\|(I-T) x_{n}\right\|+\left(\left\|(I-T) x_{n}\right\|+\mu_{n}^{(1)} \phi\left(\left\|(I-T) x_{n}\right\|\right)\right. \\
& \left.+\xi_{n}^{(1)}\right)+\left(\left\|(I-T) x_{n}\right\|+\mu_{n}^{(2)} \phi\left(\left\|(I-T) x_{n}\right\|\right)+\xi_{n}^{(2)}\right) \\
& +\left(\left\|(I-T) x_{n}\right\|+\mu_{n}^{(3)} \phi\left(\left\|(I-T) x_{n}\right\|\right)+\xi_{n}^{(3)}\right) \\
& +\cdots+\left(\left\|(I-T) x_{n}\right\|+\mu_{n}^{(j-1)} \phi\left(\left\|(I-T) x_{n}\right\|\right)+\xi_{n}^{(j-1)}\right) \\
= & \left\|(I-T) x_{n}\right\|+\sum_{j=1}^{m-1}\left\|(I-T) x_{n}\right\|+\sum_{j=1}^{m-1} \mu_{n}^{(j)} \phi\left(\left\|(I-T) x_{n}\right\|\right) \\
& +\sum_{j=1}^{m-1} \xi_{n}^{(j)} \\
\leq & m\left\|x_{n}-T x_{n}\right\|+m \mu_{n} \phi\left(\left\|(I-T) x_{n}\right\|\right)+m \xi_{n},
\end{aligned}
$$

where $\mu_{n}=\max _{1 \leq j \leq m-1}\left\{\mu_{n}^{(j)}\right\}$ and $\xi_{n}=\max _{1 \leq j \leq m-1}\left\{\xi_{n}^{(j)}\right\}$.

From (3.2) and (3.3), we get

$$
\left\|x_{n}-T(P T)^{j-1} x_{n}\right\|<\epsilon
$$

Now, since $T: K \longrightarrow E$ is $L$-Lipschitizian and total asymptotically nonexpansive, so is $T: C \longrightarrow$ $E$. Therefore, $\forall j \geq 1, T(P T)^{j-1}: C \longrightarrow E$ is Lipschitizian mapping with the Lipschitz constant $\mu_{j} \geq 1$. 
In addition,

$$
\begin{aligned}
\left\|T(P T)^{j-1} y_{n}-y_{n}\right\|= & \| T(P T)^{j-1} y_{n}-\sum_{i=1}^{m(n)} t_{i}^{(n)} T(P T)^{j-1} x_{i+n}+\sum_{i=1}^{m(n)} t_{i}^{(n)} T(P T)^{j-1} x_{i+n} \\
& -\sum_{i=1}^{m(n)} t_{i}^{(n)} x_{i+n} \| \\
\leq & \left\|T(P T)^{j-1} y_{n}-\sum_{i=1}^{m(n)} t_{i}^{(n)} T(P T)^{j-1} x_{i+n}\right\| \\
& +\sum_{i=1}^{m(n)} t_{i}^{(n)}\left\|T(P T)^{j-1} x_{i+n}-x_{i+n}\right\| .
\end{aligned}
$$

Using (3.4), we get

$$
\sum_{i=1}^{m(n)} t_{i}^{(n)}\left\|T(P T)^{j-1} x_{i+n}-x_{i+n}\right\|<\epsilon, \forall n \geq N .
$$

Furthermore, by Lemma 2.5, there exists a strictly increasing continous function $\phi:[0, \infty) \longrightarrow$ $[0, \infty)$ with $\phi(0)=0$ such that for all $n \geq N$, we have

$$
\begin{aligned}
\left\|T(P T)^{j-1} y_{n}-\sum_{i=1}^{m(n)} t_{i}^{(n)} T(P T)^{j-1} x_{i+n}\right\|= & \left\|T(P T)^{j-1}\left(\sum_{i=1}^{m(n)} t_{i}^{(n)} x_{i+n}\right)-\sum_{i=1}^{m(n)} t_{i}^{(n)} T(P T)^{j-1} x_{i+n}\right\| \\
\leq & \mu_{j} \phi^{-1}\left\{m a x _ { 1 \leq j , k \leq n } \left(\left\|x_{i+n}-x_{i+k}\right\|\right.\right. \\
& \left.\left.-\mu_{j}^{-1}\left\|T(P T)^{J-1} x_{i+n}-T(P T)^{J-1} x_{k+n}\right\|\right)\right\} \\
= & \mu_{j} \phi^{-1}\left\{\operatorname { m a x } _ { 1 \leq j , k \leq n } \left(\| x_{i+n}-T(P T)^{J-1} x_{i+n}\right.\right. \\
& +T(P T)^{J-1} x_{i+n}-T(P T)^{J-1} x_{k+n} \\
& +T(P T)^{J-1} x_{k+n}-x_{i+k} \| \\
& \left.\left.-\mu_{j}^{-1}\left\|T(P T)^{J-1} x_{i+n}-T(P T)^{J-1} x_{k+n}\right\|\right)\right\} \\
\leq & \mu_{j} \phi^{-1}\left\{m a x _ { 1 \leq j , k \leq n } \left(\left\|x_{i+n}-T(P T)^{J-1} x_{i+n}\right\|\right.\right. \\
& +\left\|T(P T)^{J-1} x_{i+n}-T(P T)^{J-1} x_{k+n}\right\| \\
& +\left\|T(P T)^{J-1} x_{k+n}-x_{i+k}\right\| \\
& \left.\left.-\mu_{j}^{-1}\left\|T(P T)^{J-1} x_{i+n}-T(P T)^{J-1} x_{k+n}\right\|\right)\right\} \\
\leq & \mu_{j} \phi^{-1}\left\{m a x _ { 1 \leq j , k \leq n } \left(\epsilon+\epsilon+\left(1-\mu_{j}^{-1}\right)\right.\right. \\
& \left.\left.\times\left\|T(P T)^{J-1} x_{i+n}-T(P T)^{J-1} x_{k+n}\right\|\right)\right\} \\
\leq & \mu_{j} \phi^{-1}\left\{m a x _ { 1 \leq j , k \leq n } \left(\epsilon+\epsilon+\left(1-\mu_{j}^{-1}\right) \mu_{j}\right.\right. \\
& \left.\times\left\|x_{i+n}-x_{k+n}\right\|\right\} \\
\leq & \mu_{j} \phi^{-1}\left\{\operatorname { m a x } _ { 1 \leq j , k \leq n } \left(\epsilon+\epsilon+\left(1-\mu_{j}^{-1}\right) \mu_{j}\right.\right. \\
\times & \times\left(\left\|x_{i+n}\right\|+\left\|x_{k+n}\right\|\right\} . \\
&
\end{aligned}
$$


Thus,

$$
\left\|T(P T)^{j-1} y_{n}-\sum_{i=1}^{m(n)} t_{i}^{(n)} T(P T)^{j-1} x_{i+n}\right\| \leq \mu_{j} \phi^{-1}\left(\epsilon+\epsilon+2 r\left(1-\mu_{j}^{-1}\right) \mu_{j}\right),
$$

since $x_{i+n}$ and $x_{k+n}$ are both in $C$.

Also, (3.5), (3.6) and (3.7) imply that

$$
\left\|T(P T)^{j-1} y_{n}-y_{n}\right\| \leq \mu_{j} \phi^{-1}\left(\epsilon+\epsilon+2 r\left(1-\mu_{j}^{-1}\right) \mu_{j}\right) .
$$

Taking $\lim \sup _{n \rightarrow \infty}$ on both sides of (3.8) and noting that $\epsilon>0$ is arbitrary, we have that

$$
\limsup _{n \rightarrow \infty}\left\|T(P T)^{j-1} y_{n}-y_{n}\right\| \leq \mu_{j} \phi^{-1}\left(2 r\left(1-\mu_{j}^{-1}\right) \mu_{j}\right) .
$$

On the other hand, for any $j \geq 1$, it follows from (3.1) that

$$
\begin{aligned}
\left\|T(P T)^{j-1} \omega-\omega\right\| & \leq\left\|T(P T)^{j-1} \omega-T(P T)^{j-1} y_{n}\right\|+\left\|T(P T)^{j-1} y_{n}-y_{n}\right\|+\left\|y_{n}-\omega\right\| \\
& \leq \mu_{j}\left\|y_{n}-\omega\right\|+\left\|T(P T)^{j-1} y_{n}-y_{n}\right\|+\left\|y_{n}-\omega\right\| .
\end{aligned}
$$

Taking lim $\sup _{n \rightarrow \infty}$ on both sides of the above inequality and using (3.1) and (3.9), we have

$$
\left\|T(P T)^{j-1} \omega-\omega\right\| \leq \mu_{j} \phi^{-1}\left(2 r\left(1-\mu_{j}^{-1}\right) \mu_{j}\right) .
$$

Again, taking $\lim \sup _{j \rightarrow \infty}$ on both sides of the above inequality, we have

$$
\limsup _{j \rightarrow \infty}\left\|T(P T)^{j-1} \omega-\omega\right\| \leq \phi^{-1}(0)=0,
$$

which implies that $\left\|T(P T)^{j-1} \omega-\omega\right\| \rightarrow 0$ as $j \rightarrow \infty$, and hence proving our claim. By continuity of $T P$, we have that

$$
\lim _{j \rightarrow \infty} T P\left(T(P T)^{j-1} \omega\right)=T P \omega=T \omega=\omega
$$

This completes the proof.

Lemma 3.2. Let $E$ be a uniformly convex Banach space and $K$ a nonempty closed convex subset of $E$. Let $S_{1}, S_{2}, S_{3}: K \longrightarrow K$ be three total asymptotically nonexpansive self mapping with sequences $\left\{k_{n}^{(1)}\right\},\left\{k_{n}^{(2)}\right\},\left\{k_{n}^{(3)}\right\} \in[1, \infty)$,

$\left\{w_{n}^{(1)}\right\},\left\{w^{(2)}\right\}_{n},\left\{w_{n}^{(3)}\right\} \in[1, \infty)$ and $T_{1}, T_{2}, T_{3}: K \longrightarrow E$ are three total asymptotically nonexpansive nonself mappings with sequences $\left\{\mu_{n}^{(1)}\right\},\left\{\mu_{n}^{(2)}\right\},\left\{\mu_{n}^{(3)}\right\} \in[1, \infty),\left\{\nu_{n}^{(1)}\right\},\left\{\nu_{n}^{(2)}\right\}$,

$\left\{\nu_{n}^{(3)}\right\} \in[1, \infty)$. Let $\left\{x_{n}\right\}$ be the sequence defined by (1.7), where $\left\{\alpha_{n}\right\}$ and $\left\{\beta_{n}\right\}$ are real sequences $\in[0,1)$. Suppose $\mathcal{F}=\left(F\left(T_{i}\right) \cap F\left(S_{i}\right)\right) \neq \emptyset$. If the following conditions hold:

i. $\sum_{n=1}^{\infty} k_{n}^{(1)}<\infty, \sum_{n=1}^{\infty} k_{n}^{(2)}<\infty, \sum_{n=1}^{\infty} k_{n}^{(3)}<\infty, \sum_{n=1}^{\infty} \mu_{n}^{(1)}<\infty, \sum_{n=1}^{\infty} \mu_{n}^{(2)}<\infty$, $\sum_{n=1}^{\infty} \mu_{n}^{(3)}<\infty, \sum_{n=1}^{\infty} \nu_{n}^{(1)}<\infty, \sum_{n=1}^{\infty} \nu_{n}^{(2)}<\infty, \sum_{n=1}^{\infty} \nu_{n}^{(3)}<\infty$,

ii. There exists a constant $M>0$ such that $\Psi(t)=\phi(t) \leq M t, t \leq 0$.

Then, $\lim _{n \infty}\left\|x_{n}-q\right\|$ and $\lim _{n \infty} d\left(x_{n}-F\right)$ both exist for all $q \in F$. 
Proof. Set $h_{n}=\max \left(k_{n}^{(1)}, k_{n}^{(2)}, k_{n}^{(3)}, \mu_{n}^{(1)}, \mu_{n}^{(2)}, \mu_{n}^{(3)}\right), M=\max \left(M_{1}, M_{2}, M_{3}, M_{4}, M_{5}, M_{6}\right)$ and $\theta_{n}=$ $\max \left(\nu_{n}^{(1)}, \nu_{n}^{(2)}, \nu_{n}^{(3)}, \omega_{n}^{(1)}, \omega_{n}^{(2)}, \omega_{n}^{(3)}\right)$. Then, $\sum_{n=1}^{\infty} h_{n}<\infty$ and $\sum_{n=1}^{\infty} \theta_{n}<\infty$. For any $q \in F$, it follows from (3.1) that

$$
\begin{aligned}
\left\|z_{n}-q\right\| \leq & \mid P\left(\left(1-\beta_{n}\right) S_{3}^{n} x_{n}+\beta_{n} T_{3}\left(P T_{3}\right)^{n-1} x_{n}\right)-P(q) \| \\
\leq & \|\left(1-\beta_{n}\right) S_{3}^{n} x_{n}+\beta_{n} T_{3}\left(P T_{3}^{n-1} x_{n}-q \|\right. \\
= & \left\|\left(1-\beta_{n}\right) S_{3}^{n} x_{n}+\beta_{n} q-q-\beta_{n} q+\beta_{n} T_{3}\left(P T_{3}\right)^{n-1} x_{n}\right\| \\
= & \left\|\left(1-\beta_{n}\right) S_{3}^{n} x_{n}-\left(1-\beta_{n}\right) q+\beta_{n}\left(T_{3}\left(P T_{3}\right)^{n-1} x_{n}-q\right)\right\| \\
= & \left\|\left(1-\beta_{n}\right)\left(S_{3}^{n} x_{n}-q\right)+\beta_{n}\left(T_{3}\left(P T_{3}\right)^{n-1} x_{n}-q\right)\right\| \\
\leq & \left(1-\beta_{n}\right)\left\|S_{3}^{n} x_{n}-q\right\|+\beta_{n}\left\|T_{3}\left(P T_{3}\right)^{n-1} x_{n}-q\right\| \\
\leq & \left(1-\beta_{n}\right)\left[\left\|x_{n}-q\right\|+k_{n}^{(3)} \Psi\left(\left\|x_{n}-q\right\|\right)+\omega_{n}^{(3)}\right]+\beta_{n}\left[\left\|x_{n}-q\right\|+\mu_{n}^{(3)} \phi\left(\left\|x_{n}-q\right\|\right)\right. \\
& \left.+\nu_{n}^{(3)}\right] \\
= & \left(1-\beta_{n}\right)\left\|x_{n}-q\right\|+\left(1-\beta_{n}\right) h_{n} \Psi\left(\left\|x_{n}-q\right\|\right)+\left(1-\beta_{n}\right) \theta_{n}+\beta_{n}\left\|x_{n}-q\right\| \\
& +\beta_{n} h_{n} \phi\left(\left\|x_{n}-q\right\|\right)+\beta_{n} \theta_{n} \\
\leq & \left(1-\beta_{n}\right)\left(1+h_{n} M_{5}\right)\left\|x_{n}-q\right\|+\beta_{n}\left(1+h_{n} M_{6}\right)\left\|x_{n}-q\right\|+\theta_{n} \\
\leq & \left(1-\beta_{n}\right)\left(1+h_{n} M\right)\left\|x_{n}-q\right\|+\beta_{n}\left(1+h_{n} M\right)\left\|x_{n}-q\right\|+\theta_{n} \\
\leq & \left(1+h_{n} M\right)\left\|x_{n}-q\right\|+\theta_{n} .
\end{aligned}
$$

Also, form (1.7), we get

$$
\begin{aligned}
\left\|y_{n}-q\right\|= & \mid P\left(\left(1-\beta_{n}\right) S_{2}^{n} x_{n}+\beta_{n} T_{2}\left(P T_{2}\right)^{n-1} z_{n}\right)-P(q) \| \\
\leq & \left\|\left(1-\beta_{n}\right) S_{2}^{n} x_{n}+\beta_{n} T_{2}\left(P T_{2}\right)^{n-1} x_{n}-q\right\| \\
= & \left\|\left(1-\beta_{n}\right) S_{2}^{n} x_{n}+\beta_{n} q-q-\beta_{n} q+\beta_{n} T_{2}\left(P T_{2}\right)^{n-1} z_{n}\right\| \\
= & \left\|\left(1-\beta_{n}\right) S_{2}^{n} x_{n}-\left(1-\beta_{n}\right) q+\beta_{n}\left(T_{2}\left(P T_{2}\right)^{n-1} z_{n}-q\right)\right\| \\
= & \left\|\left(1-\beta_{n}\right)\left(S_{2}^{n} x_{n}-q\right)+\beta_{n}\left(T_{2}\left(P T_{2}\right)^{n-1} z_{n}-q\right)\right\| \\
\leq & \left(1-\beta_{n}\right)\left\|S_{2}^{n} x_{n}-q\right\|+\beta_{n}\left\|T_{2}\left(P T_{2}\right)^{n-1} z_{n}-q\right\| \\
\leq & \left(1-\beta_{n}\right)\left[\left\|x_{n}-q\right\|+k_{n}^{(2)} \Psi\left(\left\|x_{n}-q\right\|\right)+\omega_{n}^{(2)}\right]+\beta_{n}\left[\left\|z_{n}-q\right\|+\mu_{n}^{(2)} \phi\left(\left\|x_{n}-q\right\|\right)\right. \\
& \left.+\nu_{n}^{(2)}\right] \\
= & \left(1-\beta_{n}\right)\left\|x_{n}-q\right\|+\left(1-\beta_{n}\right) h_{n} \Psi\left(\left\|x_{n}-q\right\|\right)+\left(1-\beta_{n}\right) \theta_{n}+\beta_{n}\left\|x_{n}-q\right\| \\
& +\beta_{n} h_{n} \phi\left(\left\|z_{n}-q\right\|\right)+\beta_{n} \theta_{n} \\
\leq & \left(1-\beta_{n}\right)\left(1+h_{n} M_{3}\right)\left\|x_{n}-q\right\|+\beta_{n}\left(1+h_{n} M_{4}\right)\left\|z_{n}-q\right\|+\theta_{n} \\
\leq & \left(1-\beta_{n}\right)\left(1+h_{n} M\right)\left\|x_{n}-q\right\|+\beta_{n}\left(1+h_{n} M\right)\left\|z_{n}-q\right\|+\theta_{n} .
\end{aligned}
$$


Putting (3.12) into (3.14), we have

$$
\begin{aligned}
\left\|y_{n}-q\right\| & \leq\left(1-\beta_{n}\right)\left(1+h_{n} M\right)\left\|x_{n}-q\right\|+\beta_{n}\left(1+h_{n} M\right)\left[\left(1+h_{n} M\right)\left\|x_{n}-q\right\|+\theta_{n}\right]+\theta_{n} \\
& =\left(1+h_{n} M\right)\left[\left(1-\beta_{n}\right)\left\|x_{n}-q\right\|+\beta_{n}\left(\left(1+h_{n} M\right)\left\|x_{n}-q\right\|+\theta_{n}\right)\right]+\theta_{n} \\
& \left.\left.=\left(1+h_{n} M\right)\left[\left(1-\beta_{n}+\beta_{n}+\beta_{n} h_{n} M\right)\right)\left\|x_{n}-q\right\|+\theta_{n}\right)\right]+\theta_{n} \\
& \left.\left.\left.\leq\left(1+h_{n} M\right)\left[1+h_{n} M\right)\right)\left\|x_{n}-q\right\|+\theta_{n}\right)\right]+\theta_{n} \\
& =\left(1+h_{n} M\right)^{2}\left\|x_{n}-q\right\|+\left(2+h_{n} M\right) \theta_{n} .
\end{aligned}
$$

Again, using (1.7), we have

$$
\begin{aligned}
\left\|x_{n+1}-q\right\| \leq & \mid P\left(\left(1-\alpha_{n}\right) S_{1}^{n} x_{n}+\alpha_{n} T_{1}\left(P T_{1}\right)^{n-1} y_{n}\right)-P(q) \| \\
\leq & \left\|\left(1-\alpha_{n}\right) S_{1}^{n} x_{n}+\alpha_{n} T_{1}\left(P T_{1}\right)^{n-1} y_{n}-q\right\| \\
= & \left\|\left(1-\alpha_{n}\right) S_{1}^{n} x_{n}+\alpha_{n} q-q-\alpha_{n} q+\alpha_{n} T_{1}\left(P T_{1}\right)^{n-1} y_{n}\right\| \\
= & \left\|\left(1-\alpha_{n}\right) S_{1}^{n} x_{n}-\left(1-\alpha_{n}\right) q+\alpha_{n}\left(T_{1}\left(P T_{1}\right)^{n-1} y_{n}-q\right)\right\| \\
= & \left\|\left(1-\alpha_{n}\right)\left(S_{1}^{n} x_{n}-q\right)+\alpha_{n}\left(T_{1}\left(P T_{1}\right)^{n-1} y_{n}-q\right)\right\| \\
\leq & \left(1-\alpha_{n}\right)\left\|S_{1}^{n} x_{n}-q\right\|+\alpha_{n}\left\|T_{1}\left(P T_{1}\right)^{n-1} y_{n}-q\right\| \\
\leq & \left(1-\alpha_{n}\right)\left[\left\|x_{n}-q\right\|+k_{n}^{(1)} \Psi\left(\left\|x_{n}-q\right\|\right)+\omega_{n}^{(1)}\right]+\alpha_{n}\left[\left\|y_{n}-q\right\|\right. \\
& \left.+\mu_{n}^{(1)} \phi\left(\left\|y_{n}-q\right\|\right)+\nu_{n}^{(1)}\right] \\
\leq & \left(1-\alpha_{n}\right)\left\|x_{n}-q\right\|+\left(1-\alpha_{n}\right) h_{n} \Psi\left(\left\|x_{n}-q\right\|\right)+\left(1-\alpha_{n}\right) \theta_{n}+\alpha_{n}\left\|y_{n}-q\right\| \\
& +\alpha_{n} h_{n} \phi\left(\left\|y_{n}-q\right\|\right)+\alpha_{n} \theta_{n} \\
\leq & \left(1-\alpha_{n}\right)\left(1+h_{n} M_{1}\right)\left\|x_{n}-q\right\|+\alpha_{n}\left(1+h_{n} M_{2}\right)\left\|y_{n}-q\right\|+\theta_{n} \\
\leq & \left(1-\alpha_{n}\right)\left(1+h_{n} M\right)\left\|x_{n}-q\right\|+\alpha_{n}\left(1+h_{n} M\right)\left\|y_{n}-q\right\|+\theta_{n} .
\end{aligned}
$$

Putting (3.15) into (3.17), we obtain

$$
\begin{aligned}
\left\|x_{n+1}-q\right\| \leq & \left(1-\alpha_{n}\right)\left(1+h_{n} M\right)\left\|x_{n}-q\right\|+\alpha_{n}\left(1+h_{n} M\right)\left[\left(1+h_{n} M\right)^{2}\left\|x_{n}-q\right\|\right. \\
& \left.\left.+\left(2+h_{n} M\right) \theta_{n}\right]+\theta_{n}\right] \\
= & \left(1+h_{n} M\right)\left\|x_{n}-q\right\|-\alpha_{n}\left(1+h_{n} M\right)\left\|x_{n}-q\right\|+\alpha_{n}\left(1+h_{n} M\right)^{3}\left\|x_{n}-q\right\| \\
& +\alpha_{n}\left(1+h_{n} M\right)\left(2+h_{n} M\right) \theta_{n}+\theta_{n} \\
\leq & {\left[1+\left(3+3 h_{n} M+h_{n}^{2} M^{2}\right) h_{n} M\right]\left\|x_{n}-q\right\|+\left[1+\left(1+h_{n} M\right)\left(2+h_{n} M\right] \theta_{n}\right.} \\
= & \left(1+\delta_{n}\right)\left\|x_{n}-q\right\|+\rho_{n} .
\end{aligned}
$$

where $\delta_{n}=1+\left(3+3 h_{n} M+h_{n}^{2} M^{2}\right) h_{n} M$ and $\rho_{n}=\left[1+\left(1+h_{n} M\right)\left(2+h_{n} M\right] \theta_{n}\right.$. Since $\sum_{n=1}^{\infty} \delta_{n}<\infty$ and $\sum_{n=1}^{\infty} \rho_{n}<\infty$, it follows from lemma 2.1 that $\lim _{n \rightarrow \infty}\left\|x_{n}-q\right\|$ exists. 
Now taking the infimum over all $q \in F$ in (3.18), we get

$$
d\left(x_{n+1}, F\right) \leq\left(1+\delta_{n}\right) d\left(x_{n}, F\right)+\rho_{n}, \forall n \in N
$$

Again, since $\sum_{n=1}^{\infty} \delta_{n}<\infty$ and $\sum_{n=1}^{\infty} \rho_{n}<\infty$, it follows from lemma 2.1 and (3.19) that $\lim _{n \rightarrow \infty} d\left(x_{n}, F\right)$ exists. This completes the proof.

Lemma 3.3. Let $E$ be a uniformly convex Banach space and $K$ a nonempty closed convex subset of $E$. Let $S_{1}, S_{2}, S_{3}: K \longrightarrow K$ be three total asymptotically nonexpansive self mapping with sequences $\left\{k_{n}^{(1)}\right\},\left\{k_{n}^{(2)}\right\},\left\{k_{n}^{(3)}\right\} \in[1, \infty),\left\{w_{n}^{(1)}\right\},\left\{w^{(2)}\right\}_{n},\left\{w_{n}^{(3)}\right\} \in[1, \infty)$

and $T_{1}, T_{2}, T_{3}: K \longrightarrow E$ are three total asymptotically nonexpansive nonself mappings with sequences $\left\{\mu_{n}^{(1)}\right\},\left\{\mu_{n}^{(2)}\right\},\left\{\mu_{n}^{(3)}\right\} \in[1, \infty),\left\{\nu_{n}^{(1)}\right\},\left\{\nu_{n}^{(2)}\right\},\left\{\nu_{n}^{(3)}\right\} \in[1, \infty)$. Let $\left\{x_{n}\right\}$ be the sequence defined by (1.7), where $\left\{\alpha_{n}\right\}$ and $\left\{\beta_{n}\right\}$ are real sequences $\in[0,1)$. Suppose $\mathcal{F}=\left(F\left(T_{i}\right) \cap F\left(S_{i}\right)\right) \neq$ $\emptyset$. If the following conditions hold:

i. $\sum_{n=1}^{\infty} k_{n}^{(1)}<\infty, \sum_{n=1}^{\infty} k_{n}^{(2)}<\infty, \sum_{n=1}^{\infty} k_{n}^{(3)}<\infty, \sum_{n=1}^{\infty} \mu_{n}^{(1)}<\infty, \sum_{n=1}^{\infty} \mu_{n}^{(2)}<\infty$, $\sum_{n=1}^{\infty} \mu_{n}^{(3)}<\infty, \sum_{n=1}^{\infty} \nu_{n}^{(1)}<\infty, \sum_{n=1}^{\infty} \nu_{n}^{(2)}<\infty, \sum_{n=1}^{\infty} \nu_{n}^{(3)}<\infty$,

ii. $\left\|x-T_{1}\left(P T_{1}\right)^{n-1} y\right\| \leq\left\|S_{1}^{n} x-T_{1}\left(P T_{1}\right)^{n-1} y\right\|,\left\|x-T_{2}\left(P T_{2}\right)^{n-1} y\right\| \leq\left\|S_{2}^{n} x-T_{2}\left(P T_{2}\right)^{n-1} y\right\|$, $\left\|x-T_{3}\left(P T_{3}\right)^{n-1} y\right\| \leq\left\|S_{3}^{n} x-T_{3}\left(P T_{3}\right)^{n-1} y\right\|$

iii. There exists a constant $M_{1}, M_{2}>0$ such that $\Psi(t) \leq M_{1} t, \phi(t) \leq M_{2} t, t \geq 0$.

Then, $\lim _{n \infty}\left\|x_{n}-S_{i} x_{n}\right\|=0$ and $\lim _{n \infty}\left\|x_{n}-T_{i} x_{n}\right\|=0$, for $i=1,2,3$.

Proof. Set $h_{n}=\max \left(k_{n}^{(1)}, k_{n}^{(2)}, k_{n}^{(3)}, \mu_{n}^{(1)}, \mu_{n}^{(2)}, \mu_{n}^{(3)}\right), M=\max \left(M_{1}, M_{2}, M_{3}, M_{4}, M_{5}, M_{6}\right)$ and $\theta_{n}=$ $\max \left(\nu_{n}^{(1)}, \nu_{n}^{(2)}, \nu_{n}^{(3)}, \omega_{n}^{(1)}, \omega_{n}^{(2)}, \omega_{n}^{(3)}\right)$. Then, $\sum_{n=1}^{\infty} h_{n}<\infty$ and $\sum_{n=1}^{\infty} \theta_{n}<\infty$. for any given $q \in F$, $\lim _{n \infty}\left\|x_{n}-q\right\|$ exists by lemma 3.2. Now, assume that $\lim _{n \infty}\left\|x_{n}-q\right\|=c$. it follows from (3.15), (3.16) and the fact that $\sum_{n=1}^{\infty} h_{n}<\infty$ and $\sum_{n=1}^{\infty} \theta_{n}<\infty$ that

$$
\left.\lim \|\left(1-\alpha_{n}\right)\left(S_{1}^{n} x_{n}-q\right)+\alpha_{n} T_{1}\left(P T_{1}\right)^{n-1} y_{n}-q\right) \|=c
$$

Also, we have

$$
\begin{aligned}
\left\|S_{1}^{n} x_{n}-q\right\| & \leq\left\|x_{n}-q\right\|+k_{n}^{(1)} \Psi\left(\left\|x_{n}-q\right\|\right)+\omega_{n}^{(1)} \\
& \left.\leq\left\|x_{n}-q\right\|+k_{n}^{(1)} M\left\|x_{n}-q\right\|\right)+\omega_{n}^{(1)} \\
& \leq\left(1+k_{n}^{(1)} M\right)\left\|x_{n}-q\right\|+\omega_{n}^{(1)} \\
& \leq\left(1+h_{n} M\right)\left\|x_{n}-q\right\|+\theta_{n} \\
\Rightarrow \limsup \left\|S_{1}^{n} x_{n}-q\right\| & \leq \lim \sup \left[\left(1+h_{n} M\right)\left\|x_{n}-q\right\|+\theta_{n}\right]=c .
\end{aligned}
$$


Furthermore,

$$
\begin{aligned}
\left\|T_{1}\left(P T_{1}\right) y_{n}-q\right\| & \leq\left\|y_{n}-q\right\|+\mu_{n}^{(1)} \phi\left(\left\|y_{n}-q\right\|\right)+\nu_{n}^{(1)} \\
& \left.\leq\left\|y_{n}-q\right\|+\mu_{n}^{(1)} M\left\|y_{n}-q\right\|\right)+\nu_{n}^{(1)} \\
& \leq\left(1+\mu_{n}^{(1)} M\right)\left\|y_{n}-q\right\|+\nu_{n}^{(1)} \\
& \leq\left(1+h_{n} M\right)\left\|y_{n}-q\right\|+\theta_{n}
\end{aligned}
$$

Taking limsup on both sides of (3.15), we obtain

$\lim \sup \left\|y_{n}-q\right\| \leq c$ and so $\lim \sup \left\|T_{1}\left(P T_{1}\right) y_{n}-q\right\| \leq \lim \sup \left[\left(1+h_{n} M\right)\left\|y_{n}-q\right\|+\theta_{n}\right] \leq c$. Thus,

$$
\lim \sup \left\|T_{1}\left(P T_{1}\right) y_{n}-q\right\| \leq \lim \sup \left[\left(1+h_{n} M\right)\left\|y_{n}-q\right\|+\theta_{n}\right]=c .
$$

Using lemma 2.2, we get

$$
\lim _{n \rightarrow \infty}\left\|S_{1}^{n} x_{n}-T_{1}\left(P T_{1}\right)^{n-1} y_{n}\right\|=0 .
$$

By condition (ii), it follows that

$\left\|x_{n}-T_{1}\left(P T_{1}\right)^{n-1} y_{n}\right\| \leq\left\|S_{1}^{n} x_{n}-T_{1}\left(P T_{1}\right)^{n-1} y_{n}\right\|$,

and so from (3.23), we have

$$
\lim _{n \rightarrow \infty}\left\|x_{n}-T_{1}\left(P T_{1}\right)^{n-1} y_{n}\right\|=0 .
$$

Also, we have

$$
\begin{aligned}
\left\|S_{2}^{n} x_{n}-q\right\| & \leq\left\|x_{n}-q\right\|+k_{n}^{(2)} \Psi\left(\left\|x_{n}-q\right\|\right)+\omega_{n}^{(2)} \\
& \left.\leq\left\|x_{n}-q\right\|+k_{n}^{(2)} M\left\|x_{n}-q\right\|\right)+\omega_{n}^{(2)} \\
& \leq\left(1+k_{n}^{(2)} M\right)\left\|x_{n}-q\right\|+\omega_{n}^{(2)} \\
& \leq\left(1+h_{n} M\right)\left\|x_{n}-q\right\|+\theta_{n} \\
\Rightarrow \limsup \left\|S_{2}^{n} x_{n}-q\right\| & \leq \limsup \left[\left(1+h_{n} M\right)\left\|x_{n}-q\right\|+\theta_{n}\right]=c .
\end{aligned}
$$

Furthermore,

$$
\begin{aligned}
\left\|T_{2}\left(P T_{2}\right) z_{n}-q\right\| & \leq\left\|z_{n}-q\right\|+\mu_{n}^{(2)} \phi\left(\left\|z_{n}-q\right\|\right)+\nu_{n}^{(2)} \\
& \left.\leq\left\|z_{n}-q\right\|+\mu_{n}^{(2)} M\left\|z_{n}-q\right\|\right)+\nu_{n}^{(2)} \\
& \leq\left(1+\mu_{n}^{(2)} M\right)\left\|z_{n}-q\right\|+\nu_{n}^{(2)} \\
& \leq\left(1+h_{n} M\right)\left\|z_{n}-q\right\|+\theta_{n}
\end{aligned}
$$


Taking limsup on both sides of (3.12), we obtain $\limsup _{n \rightarrow \infty}\left\|z_{n}-q\right\| \leq c$ and so

$$
\lim \sup \left\|T_{2}\left(P T_{1}\right) z_{n}-q\right\| \leq \lim \sup \left[\left(1+h_{n} M\right)\left\|z_{n}-q\right\|+\theta_{n}\right] \leq c .
$$

(3.13), (3.25), (3.26) and lemma 2.2 imply

$$
\lim _{n \rightarrow \infty}\left\|S_{2}^{n} x_{n}-T_{2}\left(P T_{2}\right)^{n-1} z_{n}\right\|=0 .
$$

(3.27) and condition (ii) yields

$$
\lim _{n \rightarrow \infty}\left\|x_{n}-T_{2}\left(P T_{2}\right)^{n-1} z_{n}\right\|=0 .
$$

From (3.11), using the same argument as was used in obtaining (3.27) above, we get

$$
\lim _{n \rightarrow \infty}\left\|S_{3}^{n} x_{n}-T_{3}\left(P T_{3}\right)^{n-1} x_{n}\right\|=0
$$

Now, we prove that

$$
\lim _{n \rightarrow \infty}\left\|x_{n}-T_{1}\left(P T_{1}\right)^{n-1} x_{n}\right\|=\lim _{n \rightarrow \infty}\left\|x_{n}-T_{2}\left(P T_{2}\right)^{n-1} x_{n}\right\| \lim _{n \rightarrow \infty}\left\|x_{n}-T_{3}\left(P T_{3}\right)^{n-1} x_{n}\right\|=0 .
$$

Indeed, since $\left\|x_{n}-T_{3}\left(P T_{3}\right)^{n-1} x_{n}\right\| \leq\left\|S_{3}^{n} x_{n}-T_{3}\left(P T_{3}\right)^{n-1} x_{n}\right\|$, (by condition (ii)), it follows from (3.29) that

$$
\lim _{n \rightarrow \infty}\left\|x_{n}-T_{3}\left(P T_{3}\right)^{n-1} x_{n}\right\|=0
$$

Since, $P\left(S^{n} x_{n}\right)=S^{n} x_{n}$ and $P: E \longrightarrow K$ is a nonexpansive retraction of $E$ onto $K$, we get

$$
\begin{aligned}
\left\|z_{n}-S_{3}^{n} x_{n}\right\| & =\left\|P\left(\left(1-\gamma_{n}\right) S_{3}^{n} x_{n}+\gamma_{n} T_{3}\left(P T_{3}\right)^{n-1} x_{n}\right)-S_{3}^{n} x_{n}\right\| \\
& \leq\left\|\left(1-\gamma_{n}\right) S_{3}^{n} x_{n}+\gamma_{n} T_{3}\left(P T_{3}\right)^{n-1} x_{n}-S_{3}^{n} x_{n}\right\| \\
& =\left\|-\gamma_{n}\left(S_{3}^{n} x_{n}-\gamma_{n} T_{3}\left(P T_{3}\right)^{n-1} x_{n}\right)\right\| \\
& =\gamma_{n}\left\|\left(S_{3}^{n} x_{n}-\gamma_{n} T_{3}\left(P T_{3}\right)^{n-1} x_{n}\right)\right\|,
\end{aligned}
$$

which by (3.29) gives

$$
\lim _{n \rightarrow \infty}\left\|z_{n}-S_{3}^{n} x_{n}\right\|=0
$$

Observe that

$$
\begin{aligned}
\left\|z_{n}-x_{n}\right\|= & \left\|z_{n}-S_{3}^{n} x_{n}+S_{3}^{n} x_{n}-T_{3}\left(P T_{3}\right)^{n-1} x_{n}+T_{3}\left(P T_{3}\right)^{n-1} x_{n}-x_{n}\right\| \\
\leq & \left\|z_{n}-S_{3}^{n} x_{n}\right\|+\left\|S_{3}^{n} x_{n}-T_{3}\left(P T_{3}\right)^{n-1} x_{n}\right\| \\
& +\left\|T_{3}\left(P T_{3}\right)^{n-1} x_{n}-x_{n}\right\| .
\end{aligned}
$$

Thus, it follows from (3.29), (3.30),(3.31) and (3.32) that

$$
\lim _{n \rightarrow \infty}\left\|z_{n}-x_{n}\right\|=0
$$


Again, observe that

$$
\begin{aligned}
\left\|S_{2}^{n} x_{n}-T_{2}\left(P T_{2}\right)^{n-1} x_{n}\right\| & \leq\left\|S_{2}^{n} x_{n}-T_{2}\left(P T_{2}\right)^{n-1} z_{n}\right\|+\left\|T_{2}\left(P T_{2}\right)^{n-1} z_{n}-T_{2}\left(P T_{2}\right)^{n-1} x_{n}\right\| \\
& \leq\left\|S_{2}^{n} x_{n}-T_{2}\left(P T_{2}\right)^{n-1} z_{n}\right\|+\left(\left\|z_{n}-x_{n}\right\|+k_{n}^{(2)} \phi\left(\left\|z_{n}-x_{n}\right\|\right)+\nu_{n}^{(2)}\right. \\
& \leq\left\|S_{2}^{n} x_{n}-T_{2}\left(P T_{2}\right)^{n-1} z_{n}\right\|+\left\|z_{n}-x_{n}\right\|+M h_{n}\left(\left\|z_{n}-x_{n}\right\|\right)+\theta_{n} \\
& =\left\|S_{2}^{n} x_{n}-T_{2}\left(P T_{2}\right)^{n-1} z_{n}\right\|+\left(1+M h_{n}\right)\left\|z_{n}-x_{n}\right\|+\theta_{n} .
\end{aligned}
$$

From (3.27),(3.33), (3.34) and the fact that $\sum_{n=1}^{\infty} \theta_{n}<\infty$, we get

$$
\lim _{n \rightarrow \infty}\left\|S_{2}^{n} x_{n}-T_{2}\left(P T_{2}\right)^{n-1} x_{n}\right\|=0 .
$$

Since $\left\|x_{n}-T_{2}\left(P T_{2}\right)^{n-1} x_{n}\right\| \leq\left\|S_{2}^{n} x_{n}-T_{2}\left(P T_{2}\right)^{n-1} x_{n}\right\|$ (by condition (ii), it follows from (3.35) that

$$
\lim _{n \rightarrow \infty}\left\|x_{n}-T_{2}\left(P T_{2}\right)^{n-1} x_{n}\right\|=0 .
$$

Also, since $P\left(S^{n} x_{n}\right)=S^{n} x_{n}$ and $P: E \longrightarrow K$ is a nonexpansive retraction of $E$ onto $K$, we get

$$
\begin{aligned}
\left\|y_{n}-S_{2}^{n} x_{n}\right\| & =\left\|P\left(\left(1-\beta_{n}\right) S_{2}^{n} x_{n}+\beta_{n} T_{2}\left(P T_{2}\right)^{n-1} z_{n}\right)-S_{2}^{n} x_{n}\right\| \\
& \leq\left\|\left(1-\beta_{n}\right) S_{2}^{n} x_{n}+\beta_{n} T_{2}\left(P T_{2}\right)^{n-1} z_{n}-S_{2}^{n} x_{n}\right\| \\
& =\left\|-\beta_{n}\left(S_{2}^{n} x_{n}-\beta_{n} T_{2}\left(P T_{2}\right)^{n-1} z_{n}\right)\right\| \\
& =\beta_{n}\left\|\left(S_{2}^{n} x_{n}-\beta_{n} T_{2}\left(P T_{2}\right)^{n-1} z_{n}\right)\right\|,
\end{aligned}
$$

which by (3.27) gives

$$
\lim _{n \rightarrow \infty}\left\|y_{n}-S_{2}^{n} x_{n}\right\|=0
$$

Moreover, since

$$
\begin{aligned}
\left\|y_{n}-x_{n}\right\| & =\left\|y_{n}-S_{2}^{n} x_{n}+S_{2}^{n} x_{n}-T_{2}\left(P T_{2}\right)^{n-1} z_{n}+T_{2}\left(P T_{2}\right)^{n-1} x_{n}-z_{n}\right\| \\
& \leq\left\|y_{n}-S_{2}^{n} x_{n}\right\|+\left\|S_{2}^{n} x_{n}-T_{2}\left(P T_{2}\right)^{n-1} z_{n}\right\|+\left\|T_{2}\left(P T_{2}\right)^{n-1} z_{n}-x_{n}\right\|
\end{aligned}
$$

it follows from (3.27), (3.28) and (3.37) that

$$
\lim _{n \rightarrow \infty}\left\|y_{n}-x_{n}\right\|=0
$$

Observe that

$$
\begin{aligned}
\left\|S_{1}^{n} x_{n}-T_{1}\left(P T_{1}\right)^{n-1} x_{n}\right\| & \leq\left\|S_{1}^{n} x_{n}-T_{1}\left(P T_{1}\right)^{n-1} y_{n}\right\|+\left\|T_{1}\left(P T_{1}\right)^{n-1} y_{n}-T_{1}\left(P T_{1}\right)^{n-1} x_{n}\right\| \\
& \leq\left\|S_{1}^{n} x_{n}-T_{1}\left(P T_{1}\right)^{n-1} y_{n}\right\|+\left(\left\|y_{n}-x_{n}\right\|+k_{n}^{(1)} \Psi\left(\left\|y_{n}-x_{n}\right\|\right)+\nu_{n}^{(1)}\right. \\
& \leq\left\|S_{2}^{n} x_{n}-T_{2}\left(P T_{2}\right)^{n-1} z_{n}\right\|+\left\|y_{n}-x_{n}\right\|+M h_{n}\left(\left\|z_{n}-x_{n}\right\|\right)+\theta_{n} \\
& =\left\|S_{1}^{n} x_{n}-T_{1}\left(P T_{1}\right)^{n-1} y_{n}\right\|+\left(1+M h_{n}\right)\left\|y_{n}-x_{n}\right\|+\theta_{n} .
\end{aligned}
$$

From (3.23), (3.38), (3.39) and the fact that $\sum_{n=1}^{\infty} \theta_{n}<\infty$

$$
\lim _{n \rightarrow \infty}\left\|S_{1}^{n} x_{n}-T_{1}\left(P T_{1}\right)^{n-1} x_{n}\right\|=0 .
$$


Now, since $\left\|x_{n}-T_{1}\left(P T_{1}\right)^{n-1} x_{n}\right\| \leq\left\|S_{1}^{n} x_{n}-T_{1}\left(P T_{1}\right)^{n-1} x_{n}\right\|$ (by condition (ii), it follows from (3.40) that

$$
\lim _{n \rightarrow \infty}\left\|x_{n}-T_{1}\left(P T_{1}\right)^{n-1} x_{n}\right\|=0
$$

From

$$
\begin{aligned}
\left\|x_{n+1}-S_{1}^{n} x_{n}\right\| & =\left\|P\left[\left(1-\alpha_{n}\right) S_{1}^{n} x_{n}+\alpha_{n} T_{1}\left(P T_{1}\right)^{n-1} y_{n}\right]-S_{1}^{n} x_{n}\right\| \\
& \leq\left\|\left(1-\alpha_{n}\right) S_{1}^{n} x_{n}+\alpha_{n} T_{1}\left(P T_{1}\right)^{n-1} y_{n}-S_{1}^{n} x_{n}\right\| \\
& \left.=\|-\alpha_{n}\left(S_{1}^{n} x_{n}-T_{1}\left(P T_{1}\right)^{n-1} y_{n}\right]\right) \| \\
& \left.=\alpha_{n} \| S_{1}^{n} x_{n}-T_{1}\left(P T_{1}\right)^{n-1} y_{n}\right] \|
\end{aligned}
$$

and (3.23), we obtain

$$
\lim _{n \rightarrow \infty}\left\|x_{n+1}-S_{1}^{n} x_{n}\right\|=0
$$

From

$$
\left\|x_{n+1}-T_{1}\left(P T_{1}\right)^{n-1} y_{n}\right\| \leq\left\|x_{n+1}-S_{1}^{n} x_{n}\right\|+\left\|S_{1}^{n} x_{n}-T_{1}\left(P T_{1}\right)^{n-1} y_{n}\right\|
$$

(3.23) and (3.42), we get

$$
\lim _{n \rightarrow \infty}\left\|x_{n+1}-T_{1}\left(P T_{1}\right)^{n-1} y_{n}\right\|=0
$$

Also, from (3.23), (3.24) and the inequality

$$
\left\|S_{1}^{n} x_{n}-x_{n}\right\| \leq\left\|S_{1}^{n} x_{n}-T_{1}\left(P T_{1}\right)^{n-1} y_{n}\right\|+\left\|T_{1}\left(P T_{1}\right)^{n-1} y_{n}-x_{n}\right\|,
$$

we have

$$
\lim _{n \rightarrow \infty}\left\|S_{1}^{n} x_{n}-x_{n}\right\|=0
$$

Again, from (3.41), (3.44) and the inequality

$$
\left\|S_{1}^{n} x_{n}-T_{2}\left(P T_{2}\right)^{n-1} x_{n}\right\| \leq\left\|S_{1}^{n} x_{n}-x_{n}\right\|+\left\|x_{n}-T_{2}\left(P T_{2}\right)^{n-1} x_{n}\right\|,
$$

we have

$$
\lim _{n \rightarrow \infty}\left\|S_{1}^{n} x_{n}-T_{2}\left(P T_{2}\right)^{n-1} x_{n}\right\|=0
$$


Since

$$
\begin{aligned}
\left\|x_{n+1}-T_{2}\left(P T_{2}\right)^{n-1} y_{n}\right\| \leq & \left\|x_{n+1}-S_{1}^{n} x_{n}\right\|+\left\|S_{1}^{n} x_{n}-T_{2}\left(P T_{2}\right)^{n-1} x_{n}\right\| \\
& +\left\|T_{2}\left(P T_{2}\right)^{n-1} x_{n}-T_{2}\left(P T_{2}\right)^{n-1} y_{n}\right\| \\
\leq & \left\|x_{n+1}-S_{1}^{n} x_{n}\right\|+\left\|S_{1}^{n} x_{n}-T_{2}\left(P T_{2}\right)^{n-1} x_{n}\right\|+\left(\left\|x_{n}-y_{n}\right\|\right. \\
& \left.+k_{n}^{(2)} \phi\left(\left\|x_{n}-y_{n}\right\|\right)+\nu_{n}^{(2)}\right) \\
\leq & \left\|x_{n+1}-S_{1}^{n} x_{n}\right\|+\left\|S_{1}^{n} x_{n}-T_{2}\left(P T_{2}\right)^{n-1} x_{n}\right\|+\left\|x_{n}-y_{n}\right\| \\
& \left.+M h_{n}\left\|x_{n}-y_{n}\right\|\right)+\theta_{n} \\
= & \left\|x_{n+1}-S_{1}^{n} x_{n}\right\|+\left\|S_{1}^{n} x_{n}-T_{2}\left(P T_{2}\right)^{n-1} x_{n}\right\| \\
& \left.+\left(1+M h_{n}\right)\left\|x_{n}-y_{n}\right\|\right)+\theta_{n},
\end{aligned}
$$

it follows from (3.38), (3.42), (3.45) and the fact that $\sum_{n=1}^{\infty} \theta_{n}<\infty$ that

$$
\lim _{n \rightarrow \infty}\left\|x_{n+1}-T_{2}\left(P T_{2}\right)^{n-1} y_{n}\right\|=0
$$

Now, from (3.30), (3.41) and the inequality

$$
\left\|S_{1}^{n} x_{n}-T_{3}\left(P T_{3}\right)^{n-1} x_{n}\right\| \leq\left\|S_{1}^{n} x_{n}-x_{n}\right\|+\left\|x_{n}-T_{3}\left(P T_{3}\right)^{n-1} x_{n}\right\|,
$$

we obtain

$$
\lim _{n \rightarrow \infty}\left\|S_{1}^{n} x_{n}-T_{3}\left(P T_{3}\right)^{n-1} x_{n}\right\|=0
$$

Since

$$
\begin{aligned}
\left\|x_{n+1}-T_{3}\left(P T_{3}\right)^{n-1} y_{n}\right\| \leq & \left\|x_{n+1}-S_{1}^{n} x_{n}\right\|+\left\|S_{1}^{n} x_{n}-T_{3}\left(P T_{3}\right)^{n-1} x_{n}\right\| \\
& +\left\|T_{3}\left(P T_{3}\right)^{n-1} x_{n}-T_{3}\left(P T_{3}\right)^{n-1} y_{n}\right\| \\
\leq & \left\|x_{n+1}-S_{1}^{n} x_{n}\right\|+\left\|S_{1}^{n} x_{n}-T_{3}\left(P T_{3}\right)^{n-1} x_{n}\right\|+\left(\left\|x_{n}-y_{n}\right\|\right. \\
& \left.+k_{n}^{(3)} \phi\left(\left\|x_{n}-y_{n}\right\|\right)+\nu_{n}^{(3)}\right) \\
\leq & \left\|x_{n+1}-S_{1}^{n} x_{n}\right\|+\left\|S_{1}^{n} x_{n}-T_{3}\left(P T_{3}\right)^{n-1} x_{n}\right\|+\left\|x_{n}-y_{n}\right\| \\
& \left.+M h_{n}\left\|x_{n}-y_{n}\right\|\right)+\theta_{n} \\
= & \left\|x_{n+1}-S_{1}^{n} x_{n}\right\|+\left\|S_{1}^{n} x_{n}-T_{3}\left(P T_{3}\right)^{n-1} x_{n}\right\| \\
& \left.+\left(1+M h_{n}\right)\left\|x_{n}-y_{n}\right\|\right)+\theta_{n}
\end{aligned}
$$

it follows from (3.38), (3.42), (3.47) and the fact that $\sum_{n=1}^{\infty} \theta_{n}<\infty$ that

$$
\lim _{n \rightarrow \infty}\left\|x_{n+1}-T_{3}\left(P T_{3}\right)^{n-1} y_{n}\right\|=0
$$


Again, since $(P T i)(P T I)^{n-2} y_{n-1}, x_{n} \in K$ for $i=1,2,3$ and $T_{1}, T_{2}, T_{3}$ are three total asymptotically nonexpansive nonself mappings, we have

$$
\begin{aligned}
\left\|T_{i}\left(P T_{i}\right)^{n-1} y_{n-1}-T_{i} x_{n}\right\|= & \left\|T_{i}\left(P T_{i}\right)\left(P T_{i}\right)^{n-2} y_{n-1}-T_{i}\left(P x_{n}\right)\right\| \\
\leq & \left\|\left(P T_{i}\right)\left(P T_{i}\right)^{n-2} y_{n-1}-P\left(x_{n}\right)\right\| \\
& +k_{n}^{(i)} \phi\left(\left\|\left(P T_{i}\right)\left(P T_{i}\right)^{n-2} y_{n-1}-P\left(x_{n}\right)\right\|\right)+\nu_{n}^{(i)} \\
\leq & \left\|\left(P T_{i}\right)\left(P T_{i}\right)^{n-2} y_{n-1}-P\left(x_{n}\right)\right\| \\
& +M h_{n}\left\|(P T i)(P T i)^{n-2} y_{n-1}-P\left(x_{n}\right)\right\|+\theta_{n} \\
= & \left(1+M h_{n}\right)\left\|\left(P T_{i}\right)\left(P T_{i}\right)^{n-2} y_{n-1}-P\left(x_{n}\right)\right\|+\theta_{n} \\
= & \left(1+M h_{n}\right)\left\|T_{i}\left(P T_{i}\right)^{n-2} y_{n-1}-x_{n}\right\|+\theta_{n} .
\end{aligned}
$$

For $i=1.2 .3$, , it follows from (3.43), (3.46) and (3.48) that

$$
\lim _{n \rightarrow \infty}\left\|T i(P T i)^{n-1} y_{n-1}-T i x_{n}\right\|=0 .
$$

Observe that

$$
\left\|x_{n+1}-y_{n}\right\| \leq\left\|x_{n+1}-T_{1}\left(P T_{1}\right)^{n-1} y_{n}\right\|+\left\|T_{1}\left(P T_{1}\right)^{n-1} y_{n}-x_{n}\right\|+\left\|x_{n}-y_{n}\right\|,
$$

so that, by (3.24), (3.38) and (3.43), we get

$$
\lim _{n \rightarrow \infty}\left\|x_{n+1}-y_{n}\right\|=0
$$

Next,observe, for $i=1,2,3$, that

$$
\begin{aligned}
\left\|x_{n}-T_{i} x_{n}\right\| \leq & \left\|x_{n}-T_{i}\left(P T_{i}\right)^{n-1} x_{n}\right\|+\left\|T_{i}\left(P T_{i}\right)^{n-1} x_{n}-T_{i}\left(P T_{i}\right)^{n-1} y_{n-1}\right\| \\
& +\left\|T_{i}\left(P T_{i}\right)^{n-1} y_{n-1}-T_{i} x_{n}\right\| \\
\leq & \left\|x_{n}-T_{i}\left(P T_{i}\right)^{n-1} x_{n}\right\|+\left[\left\|x_{n}-y_{n-1}\right\|+k_{n}^{(i)} \phi\left(\left\|x_{n}-y_{n-1}\right\|\right)\right. \\
& \left.+v_{n}^{(i)}\right]+\left\|T_{i}\left(P T_{i}\right)^{n-1} y_{n-1}-T_{i} x_{n}\right\| \\
\leq & \left\|x_{n}-T_{i}\left(P T_{i}\right)^{n-1} x_{n}\right\|+\left\|x_{n}-y_{n-1}\right\|+k_{n}^{(i)} M\left\|x_{n}-y_{n-1}\right\| \\
& +v_{n}^{(i)}+\left\|T_{i}\left(P T_{1}\right)^{n-1} y_{n-1}-T_{i} x_{n}\right\| \\
= & \left.\left\|x_{n}-T_{i}\left(P T_{i}\right)^{n-1} x_{n}\right\|+\left(1+k_{n}^{(i)} M\right)\left\|x_{n}-y_{n-1}\right\|+\nu_{n}^{(i)}\right] \\
& +\left\|T_{i}\left(P T_{i}\right)^{n-1} y_{n-1}-T_{i} x_{n}\right\| \\
\leq & \left\|x_{n}-T_{i}\left(P T_{i}\right)^{n-1} x_{n}\right\|+\max \left[\sup _{n \geq 1}\left(1+k_{n}^{(i)} M\right)\right]\left\|x_{n}-y_{n-1}\right\| \\
& \left.+\max \left[\sup _{n \geq 1}\right] \nu_{n}^{(i)}\right]+\left\|T_{i}\left(P T_{i}\right)^{n-1} y_{n-1}-T_{i} x_{n}\right\|
\end{aligned}
$$

Thus, it follows from (3.30), (3.36), (3.41), (3.50) and (3.51) that $\lim _{n \rightarrow \infty}\left\|x_{n}-T_{i} x_{n}\right\|=0$, for $i=1,2,3$. 
Finally, we prove that $\lim _{n \rightarrow \infty}\left\|x_{n}-S_{i}^{n} x_{n}\right\|=0$, for $i=1,2,3$.

Infact, by condition (ii), we have for $i=1,2,3$, that

$$
\left\|x_{n}-S_{i}^{n} x_{n} \leq\right\| x_{n}-T_{i}\left(P T_{i}\right)^{n-1} x_{n}\|+\| S_{i}^{n} x_{n}-T_{i}\left(P T_{i}\right)^{n-1} x_{n} \|
$$

Thus, it follows from (3.29), (3.30), (3.36), (3.40), (3.41) and (3.45) that

$$
\lim _{n \rightarrow \infty}\left\|x_{n}-S_{i}^{n} x_{n}\right\|=0, \text { for } i=1,2,3
$$

This completes the proof of Lemma 3.3.

Lemma 3.4. Under the assumption of Lemma 3.2, for all $p_{1}, p_{2} \in \cap_{i 1}^{3}\left(F\left(S_{i}\right) \cap F\left(T_{i}\right)\right)$, the limit $\lim _{n \rightarrow \infty}\left\|x_{n}+(1-t) p_{1}-p_{2}\right\|$ exists for all $t \in[0,1]$, where $\left\{x_{n}\right\}$ is the sequence defined by (1.7).

Proof. By lemma 3.2, $\lim _{n \rightarrow \infty}\left\|x_{n}-q\right\|$ exists for all $q \in F$ and therefor $\left\{x_{n}\right\}$ is bounded. Let $a_{n}(t)=\left\|x_{n}+(1-t) p_{1}-p_{2}\right\|$ exists for all $t \in[0,1]$. Then, $\lim _{n \rightarrow \infty} a_{(0)}=\left\|p_{1}-p_{2}\right\|$ and $\lim _{n \rightarrow \infty} a_{(1)}=\left\|x_{n}-p_{2}\right\|$ exist by Lemma 3.2. It remains therefor to prove Lemma 3.4 for $t \in(0,1)$. For all $x \in K$, we define the mapping

$$
\left\{\begin{array}{l}
R_{n}(x)=P\left[\left(1-\gamma_{n}\right) S_{3}^{n}+\gamma T_{3}\left(P T_{3}\right)^{n-1} x_{n}\right] \\
W_{n}(x)=P\left[\left(1-\beta_{n}\right) S_{2}^{n}+\beta T_{2}\left(P T_{2}\right)^{n-1} x_{n}\right] \\
V_{n}(x)=P\left[\left(1-\alpha_{n}\right) S_{1}^{n}+\alpha T_{1}\left(P T_{1}\right)^{n-1} x_{n}\right], n \geq 1
\end{array}\right.
$$

Then, it follows that $x_{n+1}=V_{n} x_{n}, V_{n} p=p, \forall p \in F$. Now, from (3.12), (3.15) and (3.18) of Lemma 3.2, we see that

$$
\left\{\begin{array}{l}
\left\|R_{n(x)}-R_{n}(y)\right\| \leq\left(1+h_{n}\right) M\|x-y\|+\theta_{n} ; \\
\left\|W_{n(x)}-W_{n}(y)\right\| \leq\left(1+r_{n}\right) M\|x-y\|+\delta_{n} \theta_{n} ; \\
\left\|V_{n(x)}-V_{n}(y)\right\| \leq\left(1+e_{n}\right) M\|x-y\|+\theta_{n}=f_{n}\|x-y\|+g_{n},
\end{array}\right.
$$

where $r_{n}=2 h_{n}+h_{n}^{2} M^{2}, \delta_{n}=2+h_{n} M, e_{n}=3 h_{n} M+3 h_{n}^{2} M^{2}+h_{n}^{3} M^{3}$ and $g_{n}=\left(1+h_{n} M\right)\left(2+h_{n} M\right) \theta_{n}$ with $\sum_{n=1}^{\infty} e_{n}<\infty, \sum_{n=1}^{\infty} g_{n}<\infty$ and $f_{n}=1+e_{n}$. Since $\sum_{n=1}^{\infty} e_{n}<\infty$, it follows that $f_{n} \rightarrow 1$ as $n \rightarrow \infty$. Set

$$
\left\{\begin{array}{l}
S_{n, m}=V_{n+m-1} V_{n+m-2} \cdots V_{n}, m \in N \\
b_{n, m}=\| S_{n, m}\left(t x_{n}+(1-t) p_{1}\right)-S_{n \cdot m}\left(t x_{m}+(1-t) p_{2} \|\right.
\end{array}\right.
$$


From (3.54) and (3.55), we have

$$
\begin{aligned}
\left\|S_{n, m}(x)-S_{n, m}(y)\right\|= & \left\|V_{n+m-1} V_{n+m-2} \cdots V_{n}(x)-V_{n+m-1} V_{n+m-2} \cdots V_{n}(y)\right\| \\
\leq & f_{n+m-1}\left\|V_{n+m-2} V_{n+m-3} \cdots V_{n}(x)-V_{n+m-2} V_{n+m-3} \cdots V_{n}(y)\right\| \\
& +g_{n+m-1} \\
\leq & \left(f_{n+m-1}\right)\left(f_{n+m-2}\right) \| V_{n+m-3} V_{n+m-4} \cdots V_{n}(x) \\
& -V_{n+m-3} V_{n+m-4} \cdots V_{n}(y) \|+g_{n+m-1}+g_{n+m-2} \\
\vdots & \\
\leq & \left(\prod_{i=n}^{n+m-1} f_{i}\right)\|x-y\|+\sum_{i=n}^{n+m-1} g_{i} \\
= & B_{n}\|x-y\|+\sum_{i=n}^{n+m-1} g_{i},
\end{aligned}
$$

for all $x, y \in K$, where $B_{n}=\prod_{i=n}^{n+m-1} f_{i}, S_{n, m} x_{n}=x_{n}$ and $S_{n, m} p=p$ for all $p \in F$. Thus,

$$
\begin{aligned}
a_{n+m}(t) & =\left\|t x_{n}+(1-t) p_{1}-p_{2}\right\| \\
& =\| S_{n, m}\left(t x_{n}+(1-t) p_{1}-p_{2} \|\right. \\
& \leq b_{n, m}+\| S_{n, m}\left(t x_{n}+(1-t) p_{1}-p_{2} \| .\right.
\end{aligned}
$$

By using Theorem 2.3 in [5], we have

$$
\begin{aligned}
b_{n, m} & \leq \psi^{-1}\left(\|\left(x_{n}-u\|-\| x_{n+1}-S_{n, m} u \|\right)\right. \\
& =\psi^{-1}\left(\|\left(x_{n}-u\|-\| x_{n+1}-u+u-S_{n, m} u \|\right)\right. \\
& \leq \psi^{-1}\left(\|\left(x_{n}-u \|-\left(\left\|x_{n+1}-u\right\|+\left\|S_{n, m} u-u\right\|\right)\right),\right.
\end{aligned}
$$

so that the sequence $\left\{b_{n . m}\right\}$ converges uniformly to 0 , i.e, $b_{n, m} \rightarrow 0$ as $n \rightarrow \infty$. Since $\lim _{n \rightarrow} B_{n}=1$ and $\lim _{n \rightarrow \infty} b_{n, m}=0$, it follows from (3.57) that $\limsup _{n \rightarrow \infty} a_{n}(t) \leq \liminf _{b \rightarrow \infty} b_{n . m} \leq \liminf _{n \rightarrow \infty} a_{n}(t)$. This shows that $\lim _{n \rightarrow \infty} a_{n}(t)$ exists, i.e, $\lim _{n \rightarrow \infty}\left\|t x_{n}+(1-t) p_{1}-p_{2}\right\|$ exists for all $t \in[0,1]$. This completes the proof Lemma 3.4 .

Lemma 3.5. Under the assumption of Lemma 3.2, if $E$ has Frechet differentiable norm, then for all $p_{1}, J p_{2} \in \mathcal{F}=\cap_{i=1}^{3}\left(F\left(T_{i}\right) \cap F\left(S_{i}\right)\right)$, the $\lim _{n \rightarrow \infty}\left(\left\langle x_{n}, J\left(p_{1}-p_{2}\right)\right\rangle\right.$ exists, where $\left\{x_{n}\right\}$ is the sequence defined by (1.7). If $\omega_{\omega}\left(x_{n}\right)$ denotes the set of all weak subsequential limits of $\left\{x_{n}\right\}$, then $\left\langle q_{1}-q_{2}, J\left(p_{1}-p_{2}\right\rangle=0\right.$ for all $p_{1}, p_{2} \in F$ and for all $q_{1}, q_{2} \in \omega_{\omega}\left(x_{n}\right)$.

Proof. Suppose that $x=p_{1}-p_{2}$ with $p_{1} \neq p_{2}$ and $h=t\left(x_{n}-p_{1}\right)$ in(2.1). Then, we have

$$
\begin{aligned}
t\left(\left\langle x_{n}, J\left(p_{1}-p_{2}\right)\right\rangle+\frac{1}{2}\left\|p_{1}-p_{2}\right\|^{2}\right. & \leq \frac{1}{2}\left\|t x_{n}+(1-t) p_{1}-p_{2}\right\|^{2} \\
& \leq t\left(\left\langle x_{n}, J\left(p_{1}-p_{2}\right)\right\rangle+\frac{1}{2}\left\|p_{1}-p_{2}\right\|^{2}+b\left(t\left\|x_{n}-p_{1}\right\|\right)\right.
\end{aligned}
$$


Since $\sup _{n \geq 1}\left\|x_{n}-p\right\| \leq Q$ for some $Q>0$, we have

$$
\begin{aligned}
t \lim _{n \rightarrow \infty} \sup \left(\left\langle x_{n}, J\left(p_{1}-p_{2}\right)\right\rangle+\frac{1}{2}\left\|p_{1}-p_{2}\right\|^{2} \leq\right. & \frac{1}{2} \lim _{n \rightarrow \infty} \sup \left\|t x_{n}+(1-t) p_{1}-p_{2}\right\|^{2} \\
\leq & t \lim _{n \rightarrow \infty} \inf \left(\left\langle x_{n}, J\left(p_{1}-p_{2}\right)\right\rangle+\frac{1}{2}\left\|p_{1}-p_{2}\right\|^{2}\right. \\
& +b(t Q)
\end{aligned}
$$

That is, $t \lim _{n \rightarrow \infty} \sup \left(\left\langle x_{n}, J\left(p_{1}-p_{2}\right)\right\rangle \leq t \liminf _{n \rightarrow \infty}\left(\left\langle x_{n}, J\left(p_{1}-p_{2}\right)\right\rangle+b(t Q)\right.\right.$. If $t \rightarrow 0$, then $\lim _{n \rightarrow \infty}\left\langle x_{n}-p_{1}, J\left(p_{1}-p_{2}\right)\right\rangle$ exists for all $p_{1}, p_{2} \in F$ and for all $q_{2}, q_{2} \in \omega_{\omega}\left(x_{n}\right)$; in particular, $\left(\left\langle q_{1}-q_{2}, J\left(p_{1}-p_{2}\right)\right\rangle=0\right.$ for all $q_{2}, q_{2} \in \omega_{\omega}\left(x_{n}\right)$. This completes the proof Lemma 3.5.

Theorem 3.6. Under the assumption of Lemma 3.2, if $E$ has Frechet differentiable norm, then the sequence $\left\{x_{n}\right\}$ defined by (1.7) converges weakly to a common fixed point in $\mathcal{F}=\cap_{i=1}^{3} F\left(T_{i}\right) \cap F\left(S_{i}\right)$.

Proof. By Lemma 3.5, $\left(\left\langle q_{1}-q_{2}, J\left(p_{1}-p_{2}\right)\right\rangle=0\right.$ for all $q_{2}, q_{2} \in \omega_{\omega}\left(x_{n}\right)$. Therefore, $\left\|q^{\star}-p \star\right\|^{2}=$ $\left\langle q^{\star}-p^{\star}, J\left(q^{\star}-p^{\star}\right)\right\rangle=0$. This implies that $p^{\star}=q^{\star}$. Consequently, $\left\{x_{n}\right\}$ converges to a common fixed point of $\mathcal{F}=\cap_{i=1}^{3} F\left(T_{i}\right) \cap F\left(S_{i}\right)$. This completes the proof Theorem 3.6.

Theorem 3.7. Under the assumption of Lemma 3.2, if the dual space $E^{\star}$ of $E$ has the Kadec Klec $(K K)$ property and the mappings I $-S_{i}$ and $I-T_{i}$ for $i=1,2,3$, where I denotes the identity mapping, are demiclosed at zero, then the sequence $\left\{x_{n}\right\}$ defined by (1.7) converges weakly to a common fixed point in $\mathcal{F}=\cap_{i=1}^{3}\left(F\left(T_{i}\right) \cap F\left(S_{i}\right)\right)$.

Proof. By Lemma $3.2\left\{x_{n}\right\}$ is bounded and since $E$ is reflexive, there exists a subsequence $\left\{x_{n_{k}}\right\}$ of $\left\{x_{n}\right\}$ which converges weakly to some $q^{\star} \in K$. By Lemma 3.3, we have $\lim _{n \rightarrow \infty}\left\|x_{n_{k}}-S_{i} x_{n_{k}}\right\|=0$ and $\lim _{n \rightarrow \infty}\left\|x_{n_{k}}-T_{i} x_{n_{k}}\right\|=0$ for $i=1,2,3$. Since by hypothesis, the mappings $I-S_{i}$ and $I-T_{i}$ for $i=1,2,3$, where $I$ denotes the identity mapping, are demiclosed at zero, $S_{i} q^{\star}=q^{\star}$ and $T_{i} q^{\star}=q^{\star}$ for $i=1,2,3$; which means $q^{\star} \in \mathcal{F}=\cap_{i=1}^{3}\left(F\left(T_{i}\right) \cap F\left(S_{i}\right)\right)$. Now, we show that $\left\{x_{n}\right\}$ converges weakly to $q^{\star}$. Suppose $\left\{x_{n_{j}}\right\}$ is another subsequence of $\left\{x_{n}\right\}$ which converges weakly to $p^{\star} \in K$. By the same method as above, we have $p^{\star} \in F$ and $q^{\star} \in \omega_{\omega}\left(x_{n}\right)$. By Lemma 3.4, the limit $\lim _{n \rightarrow \infty}\left\|t x_{n}+(1-t) q^{\star}-p^{\star}\right\|$ exists for all $t \in[0,1]$ and so $q^{\star}=p^{\star}$. Thus, the sequence $\left\{x_{n}\right\}$ converges weakly to $q^{\star} \in F$. This completes the proof.

Theorem 3.8. Under the assumption of Lemma 3.2, if E satisfies Opial's condition and the mappings $I-S_{i}$ and $I-T_{i}$ for $i=1,2,3$, where I denotes the identity mapping, are demiclosed at zero, then the sequence $\left\{x_{n}\right\}$ defined by (1.7) converges weakly to a common fixed point in $\mathcal{F}=\cap_{i=1}^{3}\left(F\left(T_{i}\right) \cap\right.$ $\left.F\left(S_{i}\right)\right)$.

Proof. Let $q \star \in F$. From Lemma 3.2, the squence $\left\{\left\|x_{n}-p \star\right\|\right\}$ is convergent and hence bounded. Since, $E$ is uniformly convex, every bounded subset of $E$ is weakly compact. Thus, the exists a subsequence $\left\{x_{n_{k}}\right\}$ of $\left\{x_{n}\right\}$ which converges weakly to some $q^{\star} \in K$. By Lemma 3.3, we have 
$\lim _{n \rightarrow \infty}\left\|x_{n_{k}}-S_{i} x_{n_{k}}\right\|=0$ and $\lim _{n \rightarrow \infty}\left\|x_{n_{k}}-T_{i} x_{n_{k}}\right\|=0$ for $i=1,2,3$. Since by hypothesis, the mappings $I-S_{i}$ and $I-T_{i}$ for $i=1,2,3$, where $I$ denotes the identity mapping, are demiclosed at zero, $S_{i} q^{\star}=q^{\star}$ and $T_{i} q^{\star}=q^{\star}$ for $i=1,2$, 3.; which means $q^{\star} \in \mathcal{F}=\cap_{i=1}^{3}\left(F\left(T_{i}\right) \cap F\left(S_{i}\right)\right)$. Finally, we show that $\left\{x_{n}\right\}$ converges weakly to $q^{\star}$. Suppose on the contrary that $\left\{x_{n_{j}}\right\}$ is another subsequence of $\left\{x_{n}\right\}$ which converges weakly to $p^{\star} \in K$ and $q^{\star} \neq p^{\star}$ By Lemma 3.2, $\lim _{n \rightarrow \infty} \| x_{n}-$ $q^{\star} \|$ and $\lim _{n \rightarrow \infty}\left\|x_{n}-p^{\star}\right\|$ exist. By virtue of Opial's condition on $E$, we obtain

$$
\begin{aligned}
\lim _{n \rightarrow \infty}\left\|x_{n}-q^{\star}\right\| & =\lim _{n \rightarrow \infty}\left\|x_{n_{k}}-q^{\star}\right\| \\
& <\lim _{n \rightarrow \infty}\left\|x_{n_{k}}-p^{\star}\right\| \\
& =\lim _{n \rightarrow \infty}\left\|x_{n}-p^{\star}\right\| \\
& =\lim _{n \rightarrow \infty}\left\|x_{n_{j}}-p^{\star}\right\| \\
& <\lim _{n \rightarrow \infty}\left\|x_{n_{j}}-q^{\star}\right\| \\
& =\lim _{n \rightarrow \infty}\left\|x_{n}-q^{\star}\right\|,
\end{aligned}
$$

which is a contradiction, so $q^{\star}=p^{\star}$ Therefore, the sequence $\left\{x_{n}\right\}$ defined by (1.7) converges weakly to $q^{\star} \in F$. This completes the proof.

Corollary 3.9. Let $E$ be a uniformly convex Banach space and $K$ a nonempty closed convex subset of $E$. Let $S_{1}, S_{2}, S_{3}: K \longrightarrow K$ be three generalize asymptotically nonexpansive self mapping with sequences $\left\{k_{n}^{(1)}\right\},\left\{k_{n}^{(2)}\right\},\left\{k_{n}^{(3)}\right\} \in[1, \infty)$, $\left\{w_{n}^{(1)}\right\},\left\{w^{(2)\}_{n}},\left\{w_{n}^{(3)}\right\} \in[1, \infty)\right.$ and $T_{1}, T_{2}, T_{3}: K \longrightarrow E$ are three generalize asymptotically nonexpansive nonself mappings with sequences $\left\{\mu_{n}^{(1)}\right\},\left\{\mu_{n}^{(2)}\right\},\left\{\mu_{n}^{(3)}\right\} \in[1, \infty),\left\{\nu_{n}^{(1)}\right\},\left\{\nu_{n}^{(2)}\right\}$, $\left\{\nu_{n}^{(3)}\right\} \in[1, \infty)$. Let $\left\{x_{n}\right.$ be the sequence defined by (1.7), where $\left\{\alpha_{n}\right\}$ and $\left\{\beta_{n}\right\}$ are real sequences $\in[0,1)$.. Suppose $\mathcal{F}=\cap_{i=1}^{3}\left(F\left(T_{i}\right) \cap F\left(S_{i}\right)\right) \neq 0$. If the following conditions hold:

$$
\begin{aligned}
\text { i. } \sum_{n=1}^{\infty} k_{n}^{(1)}<\infty, \sum_{n=1}^{\infty} k_{n}^{(2)}<\infty, \sum_{n=1}^{\infty} k_{n}^{(3)}<\infty, \sum_{n=1}^{\infty} \mu_{n}^{(1)}<\infty, \sum_{n=1}^{\infty} \mu_{n}^{(2)}<\infty, \sum_{n=1}^{\infty} \mu_{n}^{(3)}< \\
\quad \infty, \sum_{n=1}^{\infty} \nu_{n}^{(1)}<\infty, \sum_{n=1}^{\infty} \nu_{n}^{(2)}<\infty, \sum_{n=1}^{\infty} \nu_{n}^{(3)}<\infty,
\end{aligned}
$$

ii. There exists a constant $M>0$ such that $\Psi(t)=\phi(t) \leq M t, t \leq 0$.

Then, $\lim _{n \infty}\left\|x_{n}-q\right\|$ and $\lim _{n \infty} d\left(x_{n}-F\right)$ both exist for all $q \in F$.

Corollary 3.10. Let $E$ be a uniformly convex Banach space and $K$ a nonempty closed convex subset of $E$. Let $S_{1}, S_{2}, S_{3}: K \longrightarrow K$ be three generalize asymptotically nonexpansive self mapping with sequences $\left\{k_{n}^{(1)}\right\},\left\{k_{n}^{(2)}\right\},\left\{k_{n}^{(3)}\right\} \in[1, \infty)$, $\left\{w_{n}^{(1)}\right\},\left\{w^{(2)}\right\}_{n},\left\{w_{n}^{(3)}\right\} \in[1, \infty)$ and $T_{1}, T_{2}, T_{3}: K \longrightarrow E$ are three generalize asymptotically nonexpansive nonself mappings with sequences $\left\{\mu_{n}^{(1)}\right\},\left\{\mu_{n}^{(2)}\right\},\left\{\mu_{n}^{(3)}\right\} \in[1, \infty),\left\{\nu_{n}^{(1)}\right\},\left\{\nu_{n}^{(2)}\right\}$, $\left\{\nu_{n}^{(3)}\right\} \in[1, \infty)$. Let $\left\{x_{n}\right\}$ be the sequence defined by (1.7), where $\left\{\alpha_{n}\right\}$ and $\left\{\beta_{n}\right\}$ are real sequences $\in[0,1)$. Suppose $\mathcal{F}=\cap_{i=1}^{3}\left(F\left(T_{i}\right) \cap F\left(S_{i}\right)\right) \neq 0$. If the following conditions hold: 
i. $\sum_{n=1}^{\infty} k_{n}^{(1)}<\infty, \sum_{n=1}^{\infty} k_{n}^{(2)}<\infty, \sum_{n=1}^{\infty} k_{n}^{(3)}<\infty, \sum_{n=1}^{\infty} \mu_{n}^{(1)}<\infty, \sum_{n=1}^{\infty} \mu_{n}^{(2)}<\infty, \sum_{n=1}^{\infty} \mu_{n}^{(3)}<$ $\infty, \sum_{n=1}^{\infty} \nu_{n}^{(1)}<\infty, \sum_{n=1}^{\infty} \nu_{n}^{(2)}<\infty, \sum_{n=1}^{\infty} \nu_{n}^{(3)}<\infty$,

ii. $\left\|x-T_{1}\left(P T_{1}\right)^{n-1} y\right\| \leq\left\|S_{1}^{n} x-T_{1}\left(P T_{1}\right)^{n-1} y\right\|,\left\|x-T_{2}\left(P T_{2}\right)^{n-1} y\right\| \leq\left\|S_{2}^{n} x-T_{2}\left(P T_{2}\right)^{n-1} y\right\|$. $\left\|x-T_{3}\left(P T_{3}\right)^{n-1} y\right\| \leq\left\|S_{3}^{n} x-T_{3}\left(P T_{3}\right)^{n-1} y\right\|$

iii. There exists a constant $M_{1}, M_{2}>0$ such that $\Psi(t) \leq M_{1} t, \phi(t) \leq M_{2} t, t \geq 0$.

Then, $\lim _{n \infty}\left\|x_{n}-S_{i} x_{n}\right\|=0$ and $\lim _{n \infty}\left\|x_{n}-T_{i} x_{n}\right\|=0$, for $i=1,2,3$.

\section{Abbreviations Used \\ Not applicable \\ Declaration:}

Availability of Data and Material

Not applicable

\section{Competing Interest}

The authors declare that there is no conflict of interest.

\section{Funding}

No specific funding received for this work

\section{Authors Contribution}

IKA and NCU wrote the paper while DII suggested the idea and did the analysis. The three authors read and approved the final manuscript.

\section{Acknowledgement}

The authors thank the anonymous reviewers for their careful reading of this paper and approved the final manuscript. 


\section{References}

[1] Ya.l. Alber, C.E. Chidume, H. Zegeye, Approximating fixed points of total asymptotically nonexpansive mappings, Fixed Point Theory Appl. 2006 (2006) 10673. https://doi .org/10.1155/FPTA/2006/10673.

[2] C.E. Chidume, E.U. Ofoedu, H. Zegeye, Strong and weak convergence theorems for asymptotically nonexpansive mappings, J. Math. Anal. Appl. 280 (2003) 364?374. https://doi .org/10.1016/S0022-247X (03)00061-1.

[3] C.E. Chidume, N. Shahzad, H. Zegeye, Convergence theorems for mappings which are asymptotically nonexpansive in the intermediate sense, Numer. Funct. Anal. Optim. 25 (2005) 239?257. https://doi.org/10.1081/ NFA- 120039611.

[4] C.E. Chidume, E.U. Ofoedu, Approximation of common fixed points for finite families of total asymptotically nonexpansive mappings, J. Math. Anal. Appl. 333 (2007) 128?141. https://doi.org/10.1016/j.jmaa.2006.09.023.

[5] J.G. Falset, W. awa Kaczor, T. Kuczumow, S. Reich, Weak convergence theorems for asymptotically nonexpansive mappings and semigroups, Nonlinear Anal.: Theory Meth. Appl. 43 (2001) 377?401. https: //doi .org/10.1016/ S0362-546X (99) 00200 - X.

[6] K. Goebel, W.A. Kirk, A fixed point theorem for asymptotically nonexpansive mappings, Proc. Amer. Math. Soc. 35 (1972) 171?171. https://doi.org/10.1090/S0002-9939-1972-0298500-3.

[7] W. Guo, W. Guo, Weak convergence theorems for asymptotically nonexpansive nonself-mappings, Appl. Math. Lett. 24 (2011) 2181?2185. https://doi.org/10.1016/j.aml.2011.06.022.

[8] W. Guo, Y.J. Cho, W. Guo, Convergence theorems for mixed type asymptotically nonexpansive mappings, Fixed Point Theory Appl. 2012 (2012) 224. https://doi.org/10.1186/1687-1812-2012-224.

[9] S.H. Khan, W. Takahashi, Approximating common fixed points of two asymptotically nonexpansive mappings, Sci. Math. Japon. 53 (2001) 143-148.

[10] Z. Opial, Weak convergence of the sequence of successive approximation for nonexpansive mappings, Bull. Amer. Math. Soc. 73 (1967) 591-597.

[11] M.O. Osilike, S.C. Aniagbosor, Weak and strong convergence theorems for fixed points of asymptotically nonexpensive mappings, Math. Computer Model. 32 (2000) 1181?1191. https://doi.org/10.1016/S0895-7177(00) 00199-0.

[12] B. E. Rhoades, Fixed point iteration for certain nonlinear mappings, J. Math. Anal. Appl., 183(1994), 118-120.

[13] J. Schu, Weak and strong convergence theorems for fixed point of asymptotically nonexpansive mappings, Bull. Austral. Math. Soc. 43 (1991), 153-159.

[14] K. Sithikul, S. Saejung, Convergence theorems for a finite family of nonexpansive and asymptotically nonexpansive mappings, Acta Univ. Palack. Olomuc. Math. 48 (2009), 139-152.

[15] W. Takahashi, G.E. Kim, Approximating fixed points of nonexpansive mappings in Banach spaces, Math. Japon. 48 (1998), 1-9.

[16] K.K. Tan, H.K. Xu, Approximating fixed point of nonexpansive mappings by the Ishikawa iteration process, J. Math. Anal. Appl. 178 (1993), 301-308.

[17] L. Wang, Strong and weak convergence theorems for common fixed points of nonself asymptotically nonexpansive mappings, J. Math. Anal. Appl. 323 (2006) 550?557. https://doi.org/10.1016/j.jmaa.2005.10.062.

[18] E. Yolacan, H. Kiziltune, On convergence theorems for total asymptotically nonexpansive nonself mappings in Banach space, J. Nonlinear Sci. Appl. 5 (2012), 389 ?402.

[19] D.I. Igbokwe, S.J. Uko, Weak and strong convergence theorems for approximating fixed points of nonexpansive mappings using composite hybrid iteration method, J. Nig. Math. Soc. 33 (2014), 129-144.

[20] D.I. Igbokwe, S.J. Uko, Weak and strong convergence of hybrid iteration methods for fixed points of asymptotically nonexpansive mappings, Adv. Fixed Point Theory. 5 (2015), 120-134.

[21] P. Wojtaszczyk, Banach space for analyst, Cambridge University Press, 1991. 